\title{
Impulse Noise Detection Techniques for Retransmission to Reduce Delay in DSL Systems
}

\author{
Dan Zhang
}

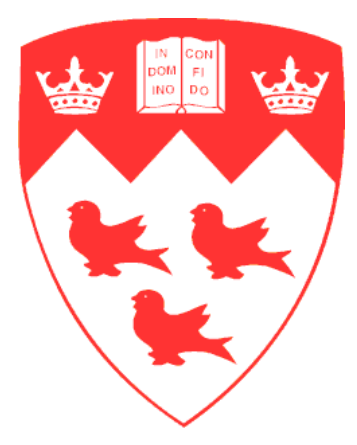

Department of Electrical and Computer Engineering

McGill University

Montreal, Canada

March 2012

A thesis submitted to McGill University in partial fulfillment of the requirements of the degree of Master of Engineering.

(C) Dan Zhang 2012 
This thesis is dedicated to my beloved parents and boyfriend 


\section{ABSTRACT}

To protect Digital Subscriber Line (DSL) systems against impulse noise (IN), interleaving combined with Reed-Solomon (RS) coding is typically deployed in the conventional DSL standards. However, interleaving introduces a long delay. To reduce such delay in conventional DSL systems that are corrupted by IN, retransmission can be used instead of interleaving. For an effective retransmission, reliable detection of corruption due to IN is required.

In this thesis, we consider three detection approaches. The first one is based on the RS decoding status since the decoder either detects the number of corrected errors or reports the failure of decoding when the errors exceed its correction capability. Retransmission is required when the transmitted codeword cannot be decoded. The second one uses the square distance method in which erasures are marked for unreliably received samples and retransmission is issued when the number of erased samples exceeds a certain threshold. Finally, the third one takes advantage of the unused tones in DSL systems in order to detect whether IN is present.

For all the above approaches, we analyze the average retransmission delay and bit error rate (BER) and provide simulation results to validate the analysis. It is found that the "Decoding Status" approach can reliably indicate received signals corrupted by IN. We consider it a trustful way to correct the symbols and detect the errors since the probability of wrong decoding for the received symbol is very low.

In the frequency selective fading channel and with the presence of Repetitive Electrical Impulse Noise (REIN), numerical results using different parameters such as various channel responses and IN powers show that retransmission offers a short delay while effectively avoiding transmission errors. Specifically, with the "Decoding Status" approach, the error caused by REIN can be completely avoided with the average retransmission delay of around $0.029 \mathrm{~ms}$ and the maximum round-trip delay of $0.75 \mathrm{~ms}$. 


\section{ABRÉGÉ}

Pour protéger les systèmes de ligne d'abonné numérique (DSL) contre le bruit impulsif (IN), les normes conventionnelles de DSL sont typiquement déployées avec de l'entrelacement combiné au codage Reed-Solomon (RS). Cependant, l'entrelacement introduit un long retard. Afin de réduire ce retard dans les systèmes conventionnels de DSL qui sont corrompus par l'IN, une retransmission peut être utilisée au lieu de l'entrelacement. Pour une retransmission efficace, une détection fiable de l'altération causée par l'IN est nécessaire.

Dans cette thèse, nous considérons trois approches de détection. La première est basée sur le statut du décodage RS puisque le décodeur détecte le nombre d'erreurs corrigées ou bien signale qu'il a échoué quand le nombre d'erreurs dépassent sa capacité de correction. Une retransmission est nécessaire quand le mot codé transmis ne peut pas être décodé. La deuxième méthode emploie la méthode des distances carrées dans laquelle les suppressions sont marquées pour les échantillons reçus non fiables et une retransmission est émise quand le nombre d'échantillons supprimés dépasse un certain seuil. Enfin, la troisième méthode est de profiter des tonalités inutilisées dans les systèmes de DSL afin de détecter si l'IN est présent.

Pour toutes les approches ci-dessus, nous analysons le retard moyen et le taux d'erreur sur les bits (BER) et nous fournissons des résultats de simulation pour valider l'analyse. Il est constaté que l'approche du statut de décodage peut indiquer de manière fiable les signaux reçus corrompus par IN et nous le considérons comme une manière efficace pour corriger les symboles et pour détecter les erreurs puisque la probabilité de mauvais décodage pour le symbole reçu est très basse.

Dans la voie sujette à évanouissement progressif de fréquences avec la présence de bruit électrique impulsif répétitif (REIN), les résultats numériques utilisant des paramètres différents tels que de diverses réponses de voies de transmission et de diverses puissances du IN prouvent que la retransmission offre un court retard tout en évitant efficacement les erreurs de transmission. Spécifiquement, avec l'approche du statut de décodage, l'erreur provoquée par le 
REIN peut complètement être évitée avec un délai de retransmission moyennant $0.029 \mathrm{~ms}$ et un retard aller-retour maximale est de $0.75 \mathrm{~ms}$. 


\section{ACKNOWLEDGEMENTS}

First I would like to express my sincere gratitude to my supervisor, Professor Tho Le-Ngoc for his encouragement and support throughout my graduate studies at McGill University. The courses taken from Professor Le-Ngoc help me with the understanding of communication systems. Every meeting with him also gives me great guidance for my research work. I also want to acknowledge the Natural Sciences and Engineering Research Council of Canada (NSERC) Grant for the financial support.

I am deeply grateful to Dr. Khuong Ho Van for helping me through my research work and providing valuable technical discussions. I would like to thank Robert Morawski for providing essential knowledge about the DSL systems and for managing the measurements on the DSL equipment.

I would also like to thank all the members of the Broadband Communications Research lab for their enthusiastic help during my study and research. I need to thank Yuxin and Yue for the great time we have both in and out of the office. I thank Danny, Leonardo and Mahsa for proofreading my thesis.

Finally, I would like to thank my parents and boyfriend, for their steady stream of encouragement and whose patient love enabled me to complete my study. 


\section{TABLE OF CONTENTS}

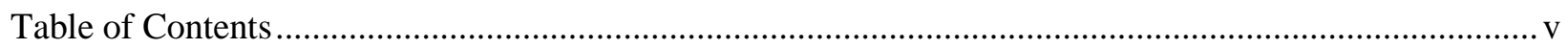

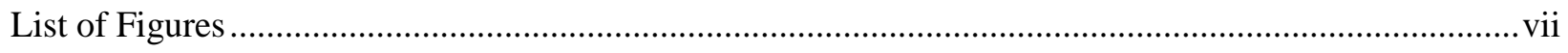

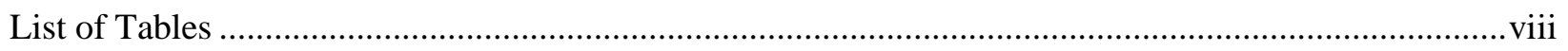

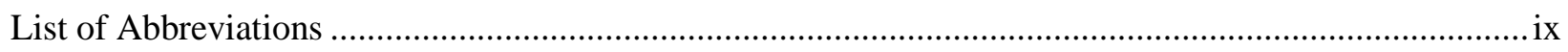

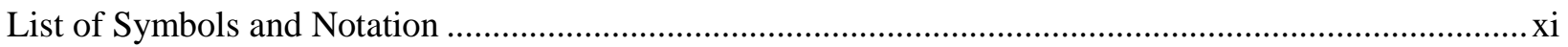

\section{Chapter 1}

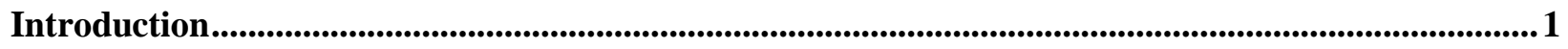

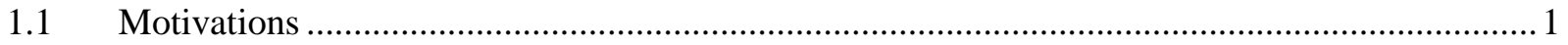

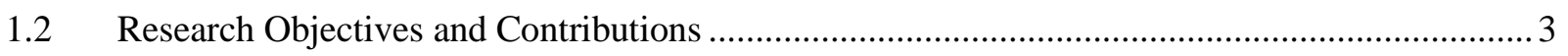

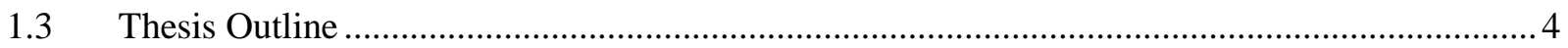

\section{Chapter 2}

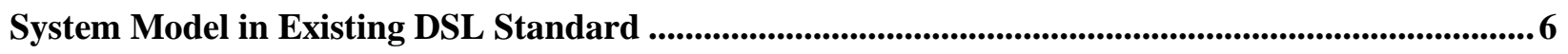

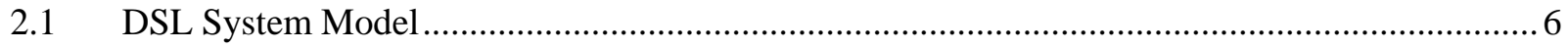

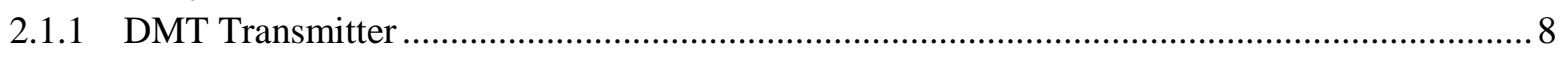

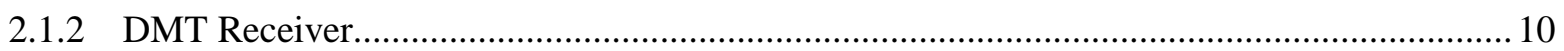

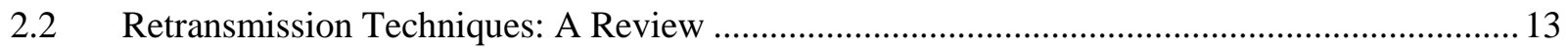

\section{Chapter 3}

Retransmission Schemes and Performance Analysis.......................................................................................15

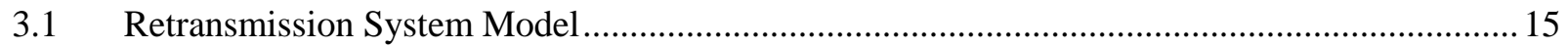

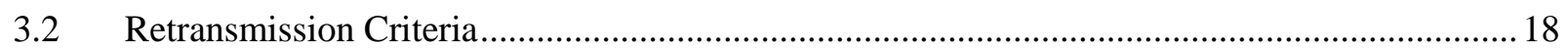

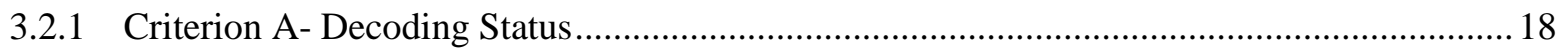

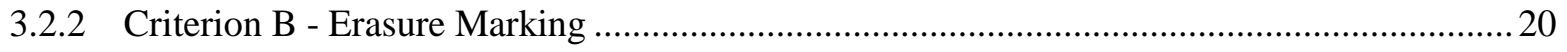

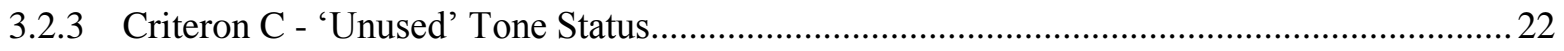

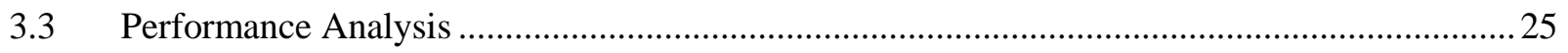

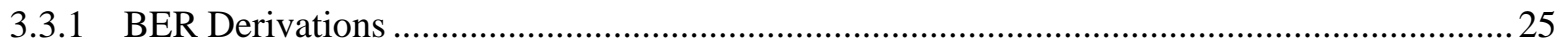

3.3.2 Performance Analysis for Criterion A - Decoding Status ................................................ 28

3.3.3 Performance Analysis for Criterion B - Erasure Marking .................................................. 32

3.3.4 Performance Analysis for Criterion C -'Unused' Tone Status............................................. 34 


\section{Chapter 4}

Performance of Proposed Retransmission Schemes: Analytical and Simulation Results...................36

4.1 Performance of proposed retransmission schemes in presence of Bernoulli-Gaussian IN ..........36

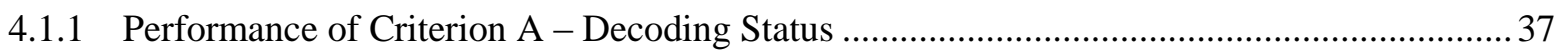

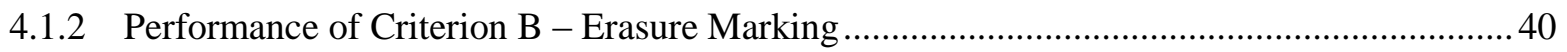

4.1.3 Performance of Criterion C -'Unused' Tone Status ............................................................. 44

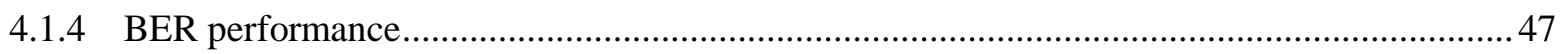

4.2 Simulation Results on Performance for REIN and measured DSL channel ...........................49

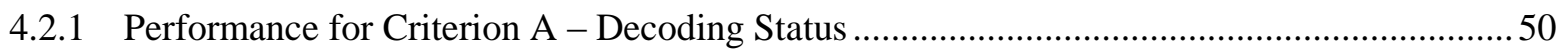

4.2.2 Performance for Criterion B - Erasure Marking................................................................ 51

4.2.3 Performance for Criterion C -'Unused' Tone Status.......................................................52

4.3 PSNR Performance of Video transmission over DSL ............................................................55

\section{Chapter 5}

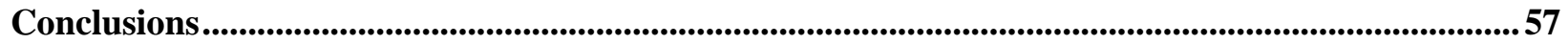

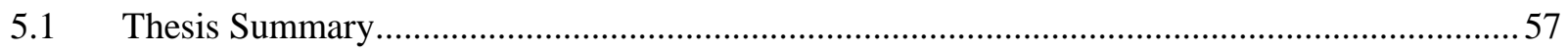

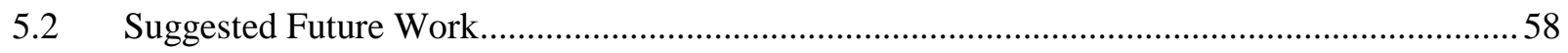




\section{LIST OF FIGURES}

Figure 1.1. From frequency-selective to a number of flat narrowband sub-carriers.................................. 2

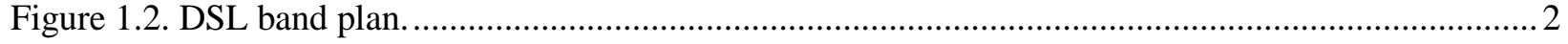

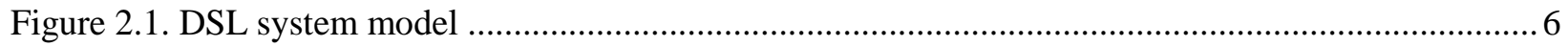

Figure 2.2. REIN model based on the standard for North America Annex A …...................................... 8

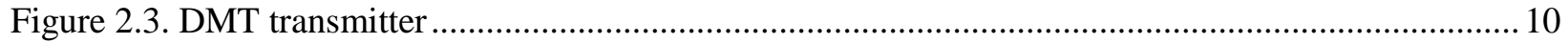

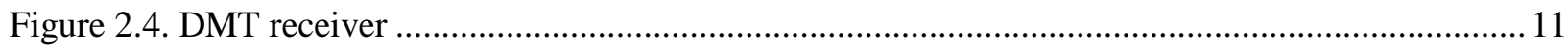

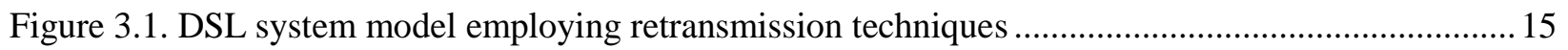

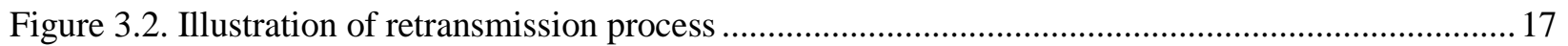

Figure 3.3. Example of retransmission scheme with buffer size of 4 DMT symbols ................................. 18

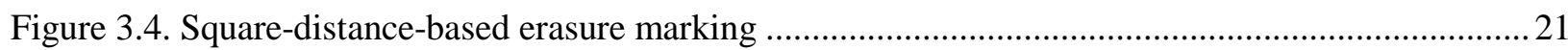

Figure 3.5. System model with retransmission and unused-tone-based IN detection...............................2 23

Figure 3.6. Codeword error rate calculation with frequency selective fading channel.............................2 27

Figure 4.1. Probabilities of decoding error and DMT symbols being decoded wrongly ........................... 38

Figure 4.2. One-time retransmission probability $\left(P_{v}, v=2\right)$ and average delay $\left(T_{\text {avg }}\right)$ versus IN PSD for

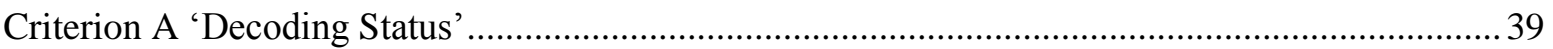

Figure 4.3. Example of square-distance threshold optimization and threshold selection versus IN PSD .. 40

Figure 4.4. Probability of miss-detection and average retransmission delay versus threshold $\beta$ for

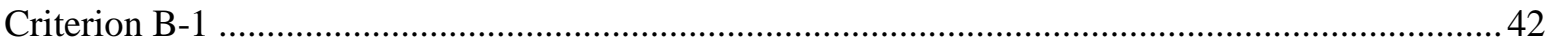

Figure 4.5. Probability of miss-detection and average retransmission delay versus threshold $\beta$ for

Criterion B-2 42

Figure 4.6. One-time retransmission probability $\left(P_{v}, v=2\right)$ and average delay $\left(T_{\text {avg }}\right)$ versus IN PSD for

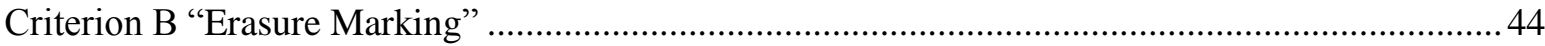

Figure 4.7. Probability of miss-detection and average retransmission delay versus $\gamma_{u} \ldots \ldots \ldots \ldots \ldots \ldots \ldots \ldots \ldots \ldots . . . . . . . . . . . .45$

Figure 4.8. One-time retransmission probability $\left(P_{v}, v=2\right)$ and average delay $\left(T_{a v g}\right)$ versus IN PSD for

Criterion C “"Unused” Tone Status”. .46

Figure 4.9. Probability of miss-detection, false alarm and average retransmission delay versus $N_{u} /$

$N_{\text {un-tones }}$

Figure 4.10. BER for a given maximum allowable number of retransmissions 48

Figure 4.11. Comparison of miss-detection, average retransmission delay and BER versus threshold $\gamma_{u} 53$ 


\section{LIST OF TABLES}

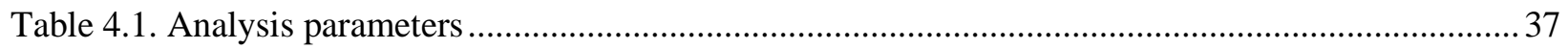

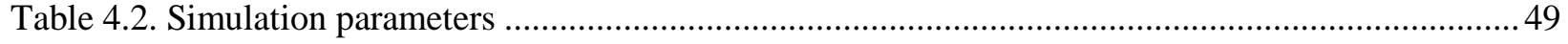

Table 4.3. Simulation results for Criterion A and REIN ......................................................................50

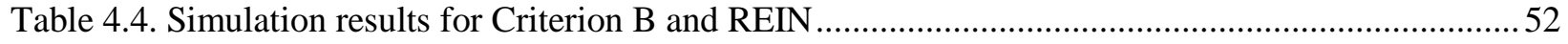

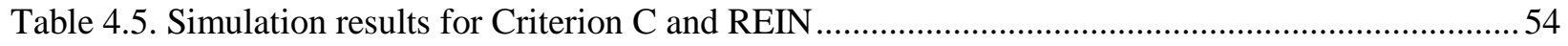

Table 4.6. PSNR of video transmission with/without REIN, with/without retransmission........................56 


\section{LIST OF ABBREVIATIONS}

\begin{tabular}{|c|c|}
\hline ADSL & Asymmetric Digital Subscriber Line \\
\hline ARQ & Automatic Repeat Request \\
\hline AWGN & Additive White Gaussian Noise \\
\hline BER & Bit Error Rate \\
\hline CRC & Cyclic Redundancy Check \\
\hline $\mathrm{CO}$ & Central Office \\
\hline DMT & Discrete Multi-Tone \\
\hline DSL & Digital Subscriber Line \\
\hline DSM & Dynamic Spectrum Management \\
\hline $\mathrm{ED}$ & Error-only Decoding \\
\hline EED & Error-and-Erasure Decoding \\
\hline FEXT & Far-End Crosstalk \\
\hline FFT & Fast Fourier Transform \\
\hline GF & Galois Field \\
\hline IFFT & Inverse Fast Fourier Transform \\
\hline IN & Impulse Noise \\
\hline INP & Impulse Noise Protection \\
\hline IPTV & Internet Protocol Television \\
\hline MSE & Mean Squared Error \\
\hline NAK & Negative Acknowledgement \\
\hline NEXT & Near-End Crosstalk \\
\hline PDF & Probability Density Function \\
\hline PDU & Protocol Data Unit \\
\hline PSD & Power Spectral Density \\
\hline PSNR & Peak Signal-to-Noise Ratio \\
\hline
\end{tabular}




$\begin{array}{ll}\text { QAM } & \text { Quadrature Amplitude Modulation } \\ \text { REIN } & \text { Repetitive Electrical Impulse Noise } \\ \text { RS } & \text { Reed-Solomon Code } \\ \text { RT } & \text { Repetition Tag } \\ \text { Rx } & \text { Receiver } \\ \text { SN } & \text { Sequence Number } \\ \text { SNR } & \text { Signal-to-Noise Ratio } \\ \text { SSM } & \text { Static Spectrum Management } \\ \text { Tx } & \text { Transmitter } \\ \text { VDSL } & \text { Very high-speed Digital Subscriber Line } \\ \text { VoIP } & \text { Voice over Internet Protocol }\end{array}$




\section{LIST OF SYMBOLS AND NOTATION}

$\alpha$

$A_{m}$

$B_{D M T}$

$B_{l, u}$

$b_{k}$

$B_{p}$

$\beta$

$c_{i}$

$c_{k} / C_{k}$

$d$

$d_{\text {min }}$

$D_{u}$

$e_{i}$

$E\left\{\left|A_{k}\right|^{2}\right\}$

$g_{k}$

$h_{k}$

$H_{o} / H_{1}$

$I(i, j)$

$i_{k} / I_{k}$

$K(i, j)$

$K_{R S} / k$

$m$

$M_{I N}$

$M_{k}$

$M A X_{I}$

Threshold of noise power outage

$M$-QAM symbol at the input of IFFT

Number of bytes in one DMT symbol

The $u^{t h}$ byte of the $l^{t h}$ codeword in a DMT symbol

A process with a sequence of zeros and ones to define the IN occupation

Number of bits per sample for the image pixels

Threshold of allowance for the erased sample percentage

Transmitted codeword

Time/frequency domain channel response

Distance of two adjacent modulated symbols

Minimum distance of Reed-Solomon code

Weight distribution for the number of decidable codeword

Error pattern added to the transmitted codeword

Signal energy per tone

White Gaussian noise as a decomposition for IN

Number of bits carried by tone $k$

Hypothesis corresponding to the event of the absence/presence of IN

Sample from the original video file

The $k^{\text {th }}$ time-domain IN sample

Sample from the recovered video file

Information length of a shortened/non-shortened Reed-Solomon codeword

Height of image in the video

Number of time-domain IN samples occurring in the DMT symbol

QAM size on the $k^{\text {th }}$ tone

Maximum possible pixel value of the video 
Width of image in the video

Discrete Fourier transform size

$N_{e}$

Number of erasures in one Reed-Solomon codeword

$N_{j}$

Number of objects with property $P(j)$

$N_{R S} / n$

Length of a shortened/non-shortened Reed-Solomon codeword

$P_{\text {cor }}$

Probability of correctly-erased samples in the "Erasure Marking" method

$P_{\text {era }}$

Probability of a Reed-Solomon symbol being erased

$P_{f}$

Probability of false alarm in the "Erasure Marking" method

$P_{m}$

Probability of miss-detection in the "Erasure Marking" method

$P_{S_{l, k}}$

Probability of symbol error

$P_{r}$

Probability of retransmission

$P_{v}$

Probability of $v$-time transmission for one DMT symbol

$P_{B_{l, u}}$

Probability of byte error

$P_{C_{i}}$

Probability of a RS codeword being successfully decoded

$P_{E_{D M T}}$

Probability of a DMT symbol being wrongly decoded

$P_{E_{i}}$

Probability of a RS codeword being wrongly decoded

$P_{F_{i}}$

Probability of a RS codeword being failed to decode

$P_{F-A}$

Probability of false alarm to request retransmission

$P_{M-D}$

Probability of miss-detection to request retransmission

$q$

Size of Galois field

$q u$

Probability that the error pattern has weight $u$

$Q$

Number of Reed-Solomon codewords in one DMT symbol

$R$

Square-distance threshold in the "Erasure Marking" method

$r_{k} / R_{k}$

The received $k^{\text {th }}$ time/frequency domain sample

$R_{k \mid H_{1}} / R_{k \mid H_{0}}$

The received signal in the hypothesis being affected/not affected by IN

$\gamma$

Ratio threshold to in the erasure marking method 


$\begin{array}{ll}\gamma_{k} & \text { SNR on } k^{t h} \text { tone } \\ s_{l, k} & \text { The } k^{t h} \text { symbol in the } l^{\text {th }} \text { codeword in a DMT symbol } \\ s_{k} / S_{k} & \text { The transmitted } k^{t h} \text { time/frequency domain sample } \\ S N R_{k} & \text { The SNR on } k^{t h} \text { tone for bit loading } \\ t & \text { Symbol-error correcting capability of RS code } \\ T & \text { Time to retransmit one DMT symbol } \\ T_{a v g} & \text { Average retransmission delay } \\ T_{p} & \text { Propagation delay } \\ T_{D M T} & \text { Duration of one DMT symbol } \\ \Gamma(\cdot) & \text { Gamma function } \\ \sigma_{G}^{2} & \text { Variance of AWGN } \\ \sigma_{i}^{2} & \text { Variance of IN } \\ u & \text { Weight of error pattern corrupted to RS codeword } \\ v_{m a x} & \text { Limitation to the buffer size of the receiver } \\ V_{n}(t) & \text { Number of vectors in a Hamming sphere with radius } t \text { in } n \text {-dimensional vector } \\ w_{k} / W_{k} & \text { The time/frequency domain AWGN sample }\end{array}$




\section{Chapter 1}

\section{Introduction}

\subsection{Motivations}

The technology of Digital Subscriber Line (DSL) brings broadband access to residence and business over ordinary telephone twisted pairs. DSL utilizes the complete bandwidth usable in the copper wires to provide high-speed transmission of hundreds of Mbps [1]. However, the telephone lines originally were not designed for high-speed data transmission and therefore introduce some factors that degrade the DSL performance.

The first problem is the high frequency selective attenuation that can cause severe inter-symbol interference in time domain at reasonably high signalling rates. To avoid frequency selectivity, DSL uses discrete multi-tone (DMT) technique to divide the transmission frequency band into a number of mutually independent smaller subcarriers (i.e., tones). As a result, DMT effectively transforms a broadband frequencyselective channel into many frequency-flat narrowband channels as shown in Figure 1.1 [2].

The second problem is the crosstalk caused by the electromagnetic interference between the telephone lines within the same bundle. Two kinds of crosstalk can exist in the bundled wires: one is near-end crosstalk (NEXT), which is the interference between the same ends of two twisted pairs; and the other one is far-end crosstalk (FEXT), which is the interference from one end of a twisted pair to the other end of the other pair. The frequency division duplex technique is used in DSL to prevent NEXT [1]. As shown in Figure 1.2, the frequency tones are divided into upstream (from user's modem towards central office) or downstream (from central office to user) transmissions on every copper pair. 


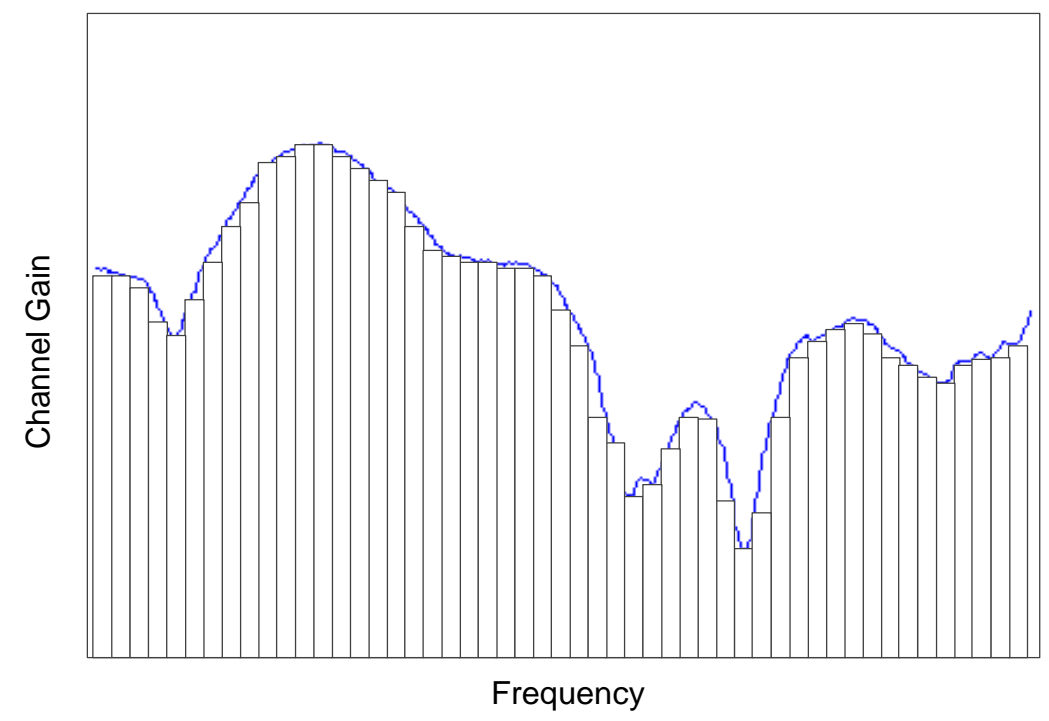

Figure 1.1. From frequency-selective to a number of flat narrowband sub-carriers

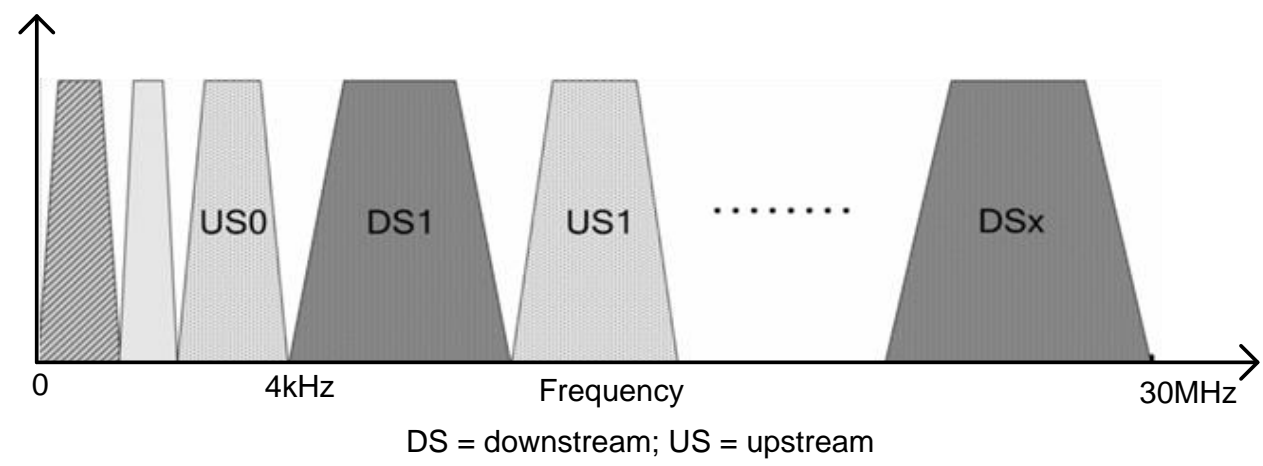

Figure 1.2. Frequency division duplex technique for DSL band plan

The background noise also impairs the transmission over the twisted pairs. The additive white Gaussian noise (AWGN) with constant spectral density and Gaussian distribution of amplitude is commonly used to model the background noise in DSL environment. AWGN comes from the thermal noise in the receiver. A suitable signal to noise ratio (SNR) margin in the bit loading procedure can effectively combat the variation of AWGN power and thus ensure reliable services in DSL systems [4]. As a result, the effect of AWGN on the system performance is negligible. However, there are other kinds of noise sources in reality. This thesis addresses the issues related to one specific source: impulse noise (IN). IN, consisting of relatively short duration "on/off" 
noise pulses, is a common kind of noise caused by a variety of sources such as switching noise, physical phenomena, home appliances, etc.[5]. IN can be tens of millivolts in amplitude and last as long as hundreds of microseconds, leading to burst errors and severely affecting high rate data transmission. Since IN power is much larger than the background noise, DSL systems cannot sustain against such high power noise given a predetermined SNR margin. Thus, IN is one of the main causes of performance degradation.

To correct the transmission errors caused by IN, Reed-Solomon (RS) coding combined with interleaving is used in the existing DSL standards [3, 6]. In particular, $\mathrm{RS}$ codes are used to correct errors in the received codewords. The interleaving technique, on the other hand, spreads error bursts over different codewords to ensure the errors in each RS codeword do not exceed its error correction capability. However, this traditional scheme has some limitations and drawbacks. For instance, a longer interleaving depth improves the performance but increases transmission latency [7]. The long delay is undesirable for applications in which the transmission requires fast interaction. As an example, the video and voice traffic types are delay sensitive since the video lag is unacceptable by most users. Therefore, retransmission techniques can be applied to replace the interleaving.

\subsection{Research Objectives and Contributions}

In this thesis, a fast retransmission technique at the physical layer is proposed for DSL in the presence of IN, aiming to reduce long transmission delay by removing interleaver from the DSL system. For an effective retransmission, the main issue is how to reliably and efficiently detect corruption due to IN for retransmission request.

In [8]-[10] various ways to apply the retransmission have been introduced. In [8], the author describes the retransmission with a real-time automatic repeat request (ARQ) protocol at the ADSL physical layer. The authors in [9] propose the forward and retransmitted systematic lossy error protection at the application layer. In reference [10], a generalized likelihood ratio test (GLRT) is derived for IN detection using unused tones. 
This thesis focuses on the retransmission criteria. Different from [8] where the cyclic redundancy check (CRC) checking result is used as a retransmission criterion and [9] where retransmission is performed at the application layer, this thesis proposes three criteria to detect errors caused by IN for retransmission request at the physical layer. The first criterion depends on the status of decoding for each codeword. The RS decoder is used to correct errors (when the number of errors in one codeword is less than its correction capability) and directly detects errors when it fails to decode. When decoding fails, the receiver issues a retransmission request through the control channel. Compared with [8] where adding CRC bits into transmitted DMT symbols results in bandwidth loss, our approach (i.e., RS code combined with retransmission) can efficiently avoid errors without the loss of transmission bandwidth.

As for the second criterion, we make use of the erasure marking technique in [11]. However, we remove the part of interleaving and propose retransmission after counting the number of erasures in each DMT symbol. When the number of erasures exceeds the given threshold, the DMT symbol is retransmitted. For the third criterion which is based on the presence of IN on unused tones, we propose an IN detector that is simpler than GLRT in [10]. Here the noise power in a DMT symbol is calculated on unused tones. If this power exceeds a certain threshold, the DMT symbol is assumed to be affected by IN and the retransmission request is then issued.

The fast retransmission technique proposed in this thesis only has the delay caused by the retransmitted DMT symbols. With certain assumptions, we derive the probability of $v$-time retransmission, average delay and BER for each of the above criteria. Simulations are taken under the same conditions to validate the analytical results.

\subsection{Thesis Outline}

The remainder of this thesis is organized as follows.

Chapter 2 introduces the existing DSL system to mitigate the effect of IN, including the procedures of bit loading, DMT modulation and demodulation. In addition, a literature review on retransmission techniques is provided. The model of retransmission system is proposed at the end of this chapter. 
Chapter 3 presents the criteria to determine when to request retransmission. Then, derivations in a simplified case with flat fading channel and Bernoulli-Gaussian IN are presented. For the first criterion, the derivation focuses on the probability of the successful decoding, the wrong decoding and the failure of decoding. After that, the average retransmission delay is computed. With regard to the second criterion, we optimize the square distance threshold by minimizing the sum of false alarm and missdetection probability, following by the calculation of average retransmission delay. Finally, the BER after decoding is computed in the last part in this chapter.

Chapter 4 simulates the DSL system model with the assumption of flat fading channel and Bernoulli-Gaussian IN. We also investigate the effect of threshold selection for "Erasure Marking" and " unused tone observation" criteria. The DSL system simulation results with frequency selective fading channel and REIN are shown, along with the PSNR test for video data transmission.

Chapter 5 summarizes the thesis, and discusses some potential subjects for future work. 


\section{Chapter 2}

\section{System Model in Existing DSL Standard}

In this chapter, we introduce the techniques in DSL system to mitigate such line impairment as attenuation and noise contamination. This chapter is organized as follows. Section 2.1 introduces the existing DSL system model which employs the RS coding/interleaving combination and figures out some drawbacks of such design. Section 2.2 describes some retransmission techniques employed in the literature to remove the interleaving/deinterleaving from the system due to the need for fast transmission.

\subsection{DSL System Model}

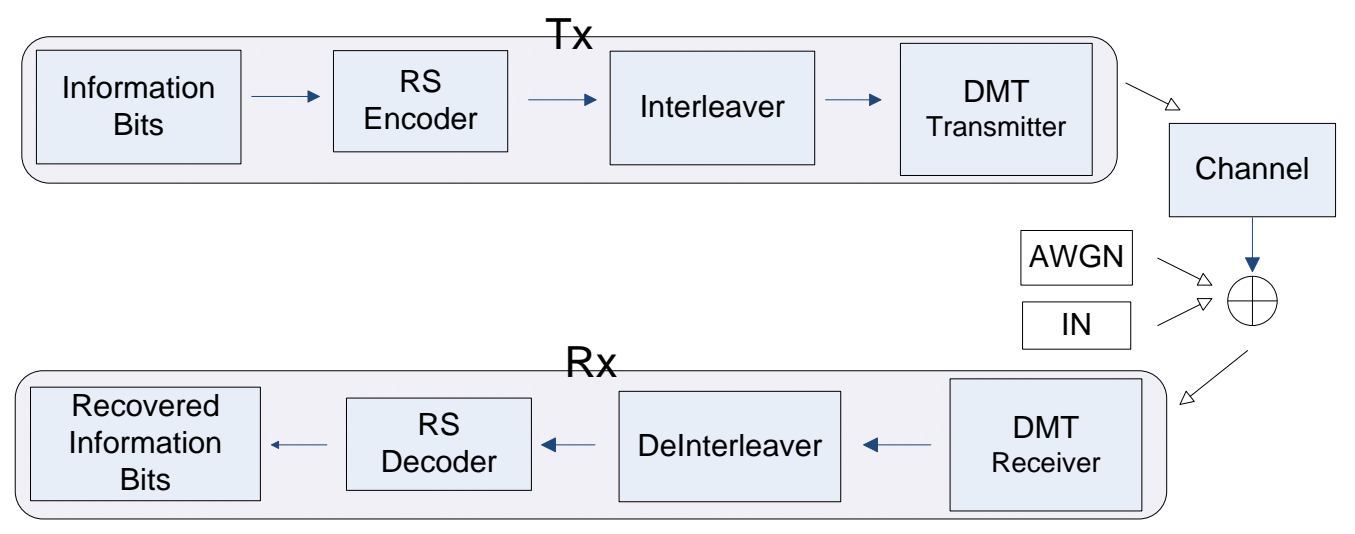

Figure 2.1. DSL system model

Figure 2.1 shows the block diagram of a DSL system using the combination of RS coding and interleaving to mitigate the effect of IN. At the transmitter, information bit sequence is first RS-encoded to generate coded bits. The coded bits are interleaved and then passed through the DMT transmitter to be modulated as DMT symbols. The resulting DMT symbols are passed through the channel and corrupted by both AWGN and IN. 
Different models of IN have been proposed in the literature to facility the analysis and practical tests of the effects of IN on DSL system performance. In our study, two IN models are considered: Bernoulli-Gaussian and Repetitive IN (REIN). It is known that IN can be characterized by the amplitude, burst duration and inter-arrival time (IAT) [12]. Modeling the statistical characteristics of IN process is difficult due to the variable and unpredictable sources. The work in [13] describes IN as a short and sharp event. Bernoulli-Gaussian IN has been widely used for the ease of analysis and demonstration. In the literature, IN is modeled as an amplitude-modulated binary-state sequence. For example, Bernoulli-Gaussian IN is a product of a Bernoulli process and a Gaussian process as $[13,24]$ :

$$
i_{k}=b_{k} g_{k}, \quad k=0,1, \ldots, N-1
$$

where $b_{k}(=0$ or 1$)$ is the Bernoulli process represented by a sequence of zeros and ones with $\operatorname{Pr}\left(b_{k}=1\right)=p$, and $g_{k}$ is a white Gaussian noise with mean zero and variance $\sigma_{i}^{2}$.

There are different IN models in the standard. Besides the IN model such as Prolonger Electrical Impulse Noise (PEIN) model, and Single High Impulse Noise (SHINE) model $[12,14]$, REIN is a model introduced in TR-114 as a standard IN type for testing in North America [15]. The REIN test uses a "burst of pseudo random AWGN" of $100 \mu$ s duration whose differential signal power spectral density is [15].

$N_{\text {REIN }}(f)=$

$\begin{cases}-116[\mathrm{dBm} / \mathrm{Hz}], & \text { for } f<2.2 \mathrm{MHz} \\ \max \left\{-116-40 \times \log _{10}\left(\frac{f}{2.2 \times 10^{6}}\right),-150\right\}[\mathrm{dBm} / \mathrm{Hz}], & \text { for } f \geq 2.2 \mathrm{MHz}\end{cases}$

It can be seen that PSD of REIN is $-116 \mathrm{dBm} / \mathrm{Hz}$ at the frequency band below $2.2 \mathrm{MHz}$ and the PSD decreases with a slope beyond 2.2 MHz. For the worst case we can assume the PSD is $-116 \mathrm{dBm} / \mathrm{Hz}$ for the entire frequency band. In time domain representation, REIN can also be represented by (2.1). However, instead of Bernoulli process, $b_{k}(=0$ or 1 ) is a periodic (or repetitive) sequence with $b_{k}=1$ during $q T$ followed by $b_{k}=0$ during $(1-q) T$ in each period $T$ where $0 \leq q \leq 1$ is the occupation rate. For example, 
the REIN model suggested for IN testing North America Annex A, Profile 8a-17a uses $T=1 /(120 \mathrm{~Hz})$ and $q=0.03$ [15]. Figure 2.2 shows the example of REIN, modeled as a periodic signal with duty cycle 0.03 and frequency of $120 \mathrm{~Hz}$ (i.e., impulse duration $q T$ $=3 \% / 120=250 \mu s)$.

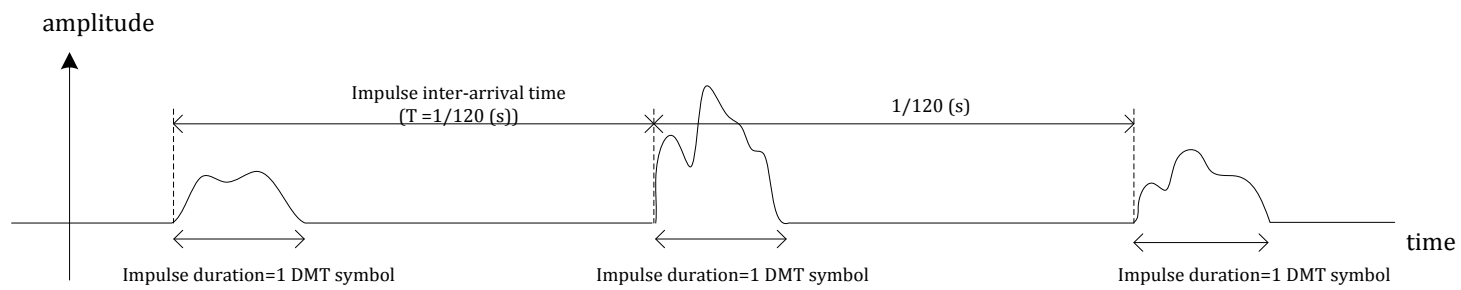

Figure 2.2. REIN model based on the standard for North America Annex A

After the transmission through the noise-corrupted channel, the received symbol is being demodulated at the DMT receiver side. Then, it is deinterleaved and RS decoded to recover the original data sequence.

In the following part we will discuss the techniques at both the DMT transmitter and receiver side in detail and explain how they mitigate the impairments in the DSL environment.

\subsubsection{DMT Transmitter}

As discussed in Chapter 1, the multicarrier DMT technique makes use of the available frequency bands on the telephone lines and splits them into many narrow-band frequency slots or tones, each can accommodate one the multiple orthogonal subcarriers. For example, in the standard VDSL2, the transmission bandwidth of $17.6 \mathrm{MHz}$ is divided into 4096 frequency tones [21]. The orthogonal sub-carriers can cope with the high frequency selectiveness in the DSL environment. The amount of bits that can be carried per sub-channel is determined by the bit loading procedure. Bit loading allows each sub-channel to carry a different number of bits adapted to the available signal to noise ratio (SNR) at that particular frequency, i.e., more bits are transmitted in subchannels with a higher SNR, while channels with lower quality carry fewer bits. Bit loading also depends on such parameters as transmit DSL profile, signal to noise ratio 
(SNR) gap, target SNR margin [6, 22], environment conditions (e.g., noise and interference). Based on the channel capacity formula, the number of bits per symbols (b/s/Hz) to be loaded in the $k^{\text {th }}$ tone is given by

$$
b_{k}=\operatorname{round}\left\{\log _{2}\left(1+\frac{S N R_{k}}{\Gamma}\right)\right\} \text {, }
$$

where round $\{\cdot\}$ represents the numerical rounding for the number of bits, $S N R_{k}$ is the signal to noise ratio on the $k^{\text {th }}$ tone which can be calculated by

$$
S N R_{k}=\left|C_{k}\right|^{2} E\left\{\left|A_{k}\right|^{2}\right\} / \sigma_{w}^{2},
$$

with $C_{k}$ representing the channel frequency response on the $k^{\text {th }}$ tone, $\mathrm{E}\left\{\left|A_{k}\right|^{2}\right\}$ is the average signal power at the transmitter, and $\sigma_{w}^{2}$ is the background noise power. $\Gamma$ is the SNR gap which is the difference between the SNR required to achieve a certain target BER with the modulation/coding and the minimum SNR required by the theoretical channel capacity [4]. In xDSL standards, the target BER of $10^{-7}$ is often used and $\Gamma=9.8+\gamma_{m}-\gamma_{c}(\mathrm{~dB})$, where $9.8 \mathrm{~dB}$ represents the required SNR gap for uncoded $M_{k^{-}}$ QAM modulation with $b_{k}=\log _{2} M_{k}$, and $\gamma_{c}$ is the coding gain for the case of embedded coding used in the $k^{t h}$ tone. The SNR margin $\gamma_{m}$ is the provided amount by which the SNR can be reduced and still maintain an operation BER at or below the target BER [22]. SNR margin is typically $6 \mathrm{~dB}$ or $9 \mathrm{~dB}$ for DSL applications in order to maintain the desired error probability under the variation of noise levels [23]. When the system is corrupted by only the background noise, with the introduced SNR margin of $6 \mathrm{~dB}$, the operation BER is $10^{-22}$. However, IN has the power much higher than background noise (i.e., REIN with PSD of $-116 \mathrm{dBm} / \mathrm{Hz}$ is $24 \mathrm{~dB}$ higher) so that provided SNR margin of 6 or $9 \mathrm{~dB}$ is not sufficient to maintain the target BER at $10^{-7}$ under the presence of IN. 


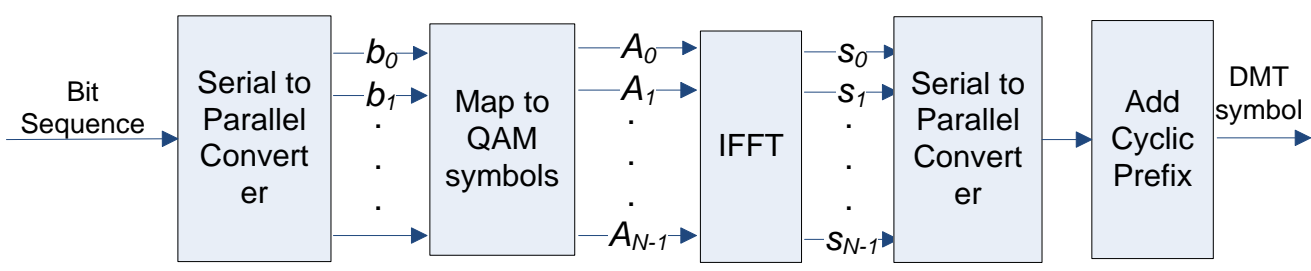

Figure 2.3. DMT transmitter

Figure 2.3 illustrates a block diagram of a DMT transmitter. Input data is first loaded to each tone with $b_{k}$ bits. After bit loading, the $b_{k}$ bits are mapped to a point in a constellation (e.g., QAM symbols). The constellation size of QAM is given by the bits loaded to each tone, i.e., for $k^{t h}$ tone which carries $b_{k}$ bits, the QAM size is $M_{k}=2^{b_{k}}$. The resulting QAM symbols are scaled to maintain a certain average power constraint. These symbols are treated as being in the frequency domain. Then, the modulated symbols are passed through an inverse fast Fourier transform (IFFT) to convert back into time domain. Finally the cyclic prefix is added. The output of the transmitter, known as a DMT symbol, is passed through the channel and corrupted by both IN and AWGN before arriving at the receiver side.

The transmitted $k^{\text {th }}$ time domain sample in one DMT symbol can be expressed as

$$
S_{k}=\frac{1}{\sqrt{N}} \sum_{m=0}^{N-1} A_{m} e^{\frac{j 2 \pi m k}{N}}, \quad k=0,1, \ldots, N-1
$$

where $N$ is the IFFT size and $A_{m}$ is the $M_{k}$-QAM symbol at the input of the IFFT.

\subsubsection{DMT Receiver}

At the receiver, the cyclic extension is removed and the Fast Fourier transform (FFT) is taken for the received symbols. The frequency domain samples are then equalized to compensate for the effect of channel response, before being demapped into the original data bits (see Figure 2.4). 


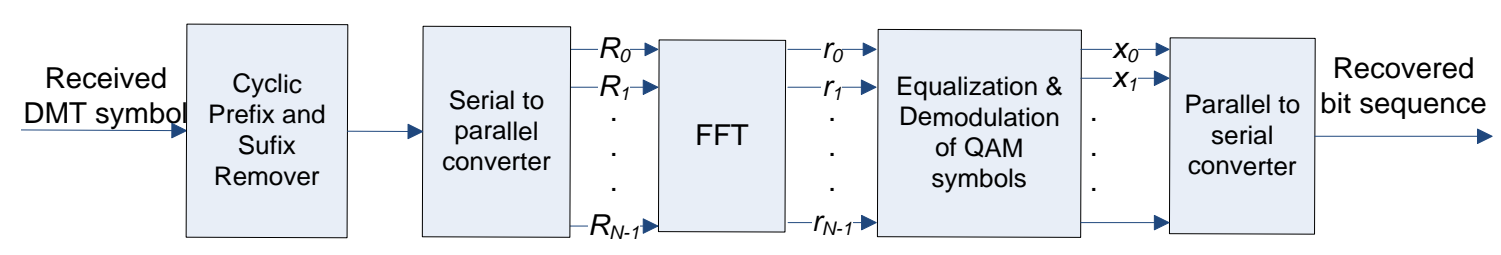

Figure 2.4. DMT receiver

The $k^{\text {th }}$ time domain sample at the receiver is written as

$$
r_{k}=(\mathbf{c} * \mathbf{s})_{k}+w_{k}+i_{k}, \quad k=0,1, \ldots, N-1
$$

where $\mathbf{c}=\left\{c_{0}, c_{1}, \ldots, c_{L-1}\right\}$ represents the channel impulse response with length $L$; $\mathbf{s}=\left\{s_{0}, s_{1}, \ldots, s_{N-1}\right\}$ is the transmitted samples with length $N$; * denotes the convolution operator; $w_{k}$ is the time domain AWGN sample with zero mean and variance of $\sigma_{G}^{2} ; i_{k}$ denotes the time domain IN sample.

The received time domain samples in the DMT symbol are converted back to frequency domain symbols by the FFT as [24]

$$
R_{k}=\frac{1}{\sqrt{N}} \sum_{m=0}^{N-1} r_{m} e^{-j 2 \pi m k / N}=C_{k} A_{k}+W_{k}+I_{k}, \quad k=0,1, \ldots, N-1
$$

where $C_{k}$ is the channel frequency response on $k^{\text {th }}$ tone and

$$
\begin{gathered}
W_{k}=\frac{1}{\sqrt{N}} \sum_{m=0}^{N-1} w_{m} e^{-j 2 \pi m k / N}, \quad k=0,1, \ldots, N-1 \\
I_{k}=\frac{1}{\sqrt{N}} \sum_{m=0}^{N-1} i_{m} e^{-j 2 \pi m k / N}, \quad k=0,1, \ldots, N-1
\end{gathered}
$$

From (2.5) and (2.7), it can be seen that even if one time-domain IN sample appears during one DMT symbol duration, the errors on consecutive tones (i.e., error burst) may occur since FFT distributes the high IN power over the whole DMT symbol. Thus, IN originated at some point in time and space can propagate through the channel to the receiver. 
As previously discussed, the target SNR margin is provided to ensure that DSL systems operate at a very low BER when only AWGN is present [24]. However, IN has much higher power than AWGN. For example, the REIN with PSD of $-116 \mathrm{dBm} / \mathrm{Hz}$ is $24 \mathrm{~dB}$ higher than AWGN with PSD of $-140 \mathrm{dBm} / \mathrm{Hz}$. As a result, DSL systems with typical SNR margin of $6 \mathrm{~dB}$ and $9 \mathrm{~dB}$ [15] cannot sustain against IN, making IN one of major causes of performance degradation.

To combat IN, the combination of RS-coding and interleaving technique is applied in the following way: at the transmitter, the input data are RS-encoded by adding parity check bytes in each codeword. The parity check bytes are used to correct the received codeword. However, the bursts of noise can cause more errors than the correction capacity in each codeword. So we need interleaving to break the burst errors into small amounts of errors. Interleaving is the method which takes data packets, dividing them into small blocks of data and then re-arranging them (interleave depth defines the number of bits in each block of data) [19]. In this way, once contiguous data are now spaced further apart into a non-continuous stream. At the receiver, data are being deinterleaved in an opposite way. In other words, the errors caused by the burst noise are now spread over different codewords. In conclusion, interleaving and deinterleaving are used to ensure the errors in each RS codeword do not exceed the correction capability.

Interleaving procedure, including the division and re-arranging of data, requires additional time which can cause a long latency. The interleave delay defines the mapping between sub-sequent input bytes at the interleave input and their placement in the bit stream at the interleave output $[19,20]$. By increasing the interleave depth, the data are divided into smaller size of blocks and the burst errors can be split into more codewords with smaller size after deinterleaving. In such way, the efficiency of RScode correction is better improved. However, the delay is longer at the same time. Specifically, a DSL system with a combination of RS coding and interleaving requires a delay of $8 \mathrm{~ms}$ to achieve the impulse noise protection $\left(\operatorname{INP}^{1}\right)$ of 2 burst errors $[15,16]$. However, some applications such as video transmission require lower latency for the

\footnotetext{
${ }^{1}$ INP is the parameter in the standard that measures the length of noise bursts, in unit of $0.25 \mathrm{~ms}$.
} 
same INP. To solve this problem, previous studies have removed the interleaving/deinterleaving from the system to fulfill the low latency requirement. The retransmission technique is proposed for such purpose - to construct an IN-combating system without interleaving.

\subsection{Retransmission Techniques: A Review}

As briefly mentioned in Chapter 1, various retransmission techniques have been suggested [8 - 10]. In [8], the author describes the retransmission with a real-time automatic repeat request (ARQ) protocol at the ADSL physical layer. In this retransmission scheme, service data units are generated in fixed time periods and passed to the transmit buffer at the ARQ sender. The scheduler takes the protocol data unit (PDU) as the transmission unit. The PDUs must contain a sequence number and a repetition tag. The received PDUs are CRC-checked by the ARQ receiver. The error free PDUs are further processed and inserted at the proper position in the receive buffer by sequence number evaluation. Lost PDUs are detected by the gaps between consecutive sequence numbers which results in the retransmission request. The advantage of this method is that it provides better error correction capability with the lower delay than the RS coding and interleaving. Nonetheless, some weaknesses of this mechanism include bandwidth loss due to the CRC bits insertion.

The authors in [9] propose the forward and retransmitted systematic lossy error protection at the application layer. In this technique, the source stream is encoded into a primary stream, which is then re-quantized and encoded to generate the redundant slices. Next, $(n, k) \mathrm{RS}$ encoding is applied across the $k$ redundant slices to generate $(n-k)$ parity slices, some of which are transmitted while the others are kept for retransmission. At the receiver, the received primary packets are used to regenerate the redundant slices. With the regenerated redundant slices and received parity slices, RS decoding is then employed. If the decoding fails, additional parity packets are requested and the decoding is attempted again to recover the missing redundant slices. The redundant slices are decoded and spliced back to the primary video stream. This method requires 
the ratio adjustment between forward and retransmitted resource allocation. Also, the extra steps of generating the redundant slices are required at the application layer.

In [10], a generalized likelihood ratio test (GLRT) is derived for IN detection using unused tones. The result of this test can be utilized as a retransmission criterion. Specifically, GLRT is based on the comparison of the estimated IN variance and the AWGN plus FEXT noise variance in the unused tones.

A simplified retransmission system is introduced in next chapter. The proposed retransmission technique does not require the $\mathrm{CRC}$-checking or the redundant slice generation. It employs a fast retransmission technique with certain criteria for the IN occupation detection. 


\section{Chapter 3}

\section{Retransmission Schemes and Performance Analysis ${ }^{2}$}

This chapter introduces the retransmission model in our study and proposes three criteria for triggering the retransmission request. BER with/without retransmission, error decoding probability, retransmission probability and the average additional delay due to retransmission are derived for each criterion.

Chapter 3 is organized as follows. Section 3.1 introduces the model of retransmission systems for protecting DSL systems against IN. Section 3.2 proposes different criteria to trigger the receiver to send the retransmission request. Section 3.3 provides performance analysis for each criterion.

\subsection{Retransmission System Model}

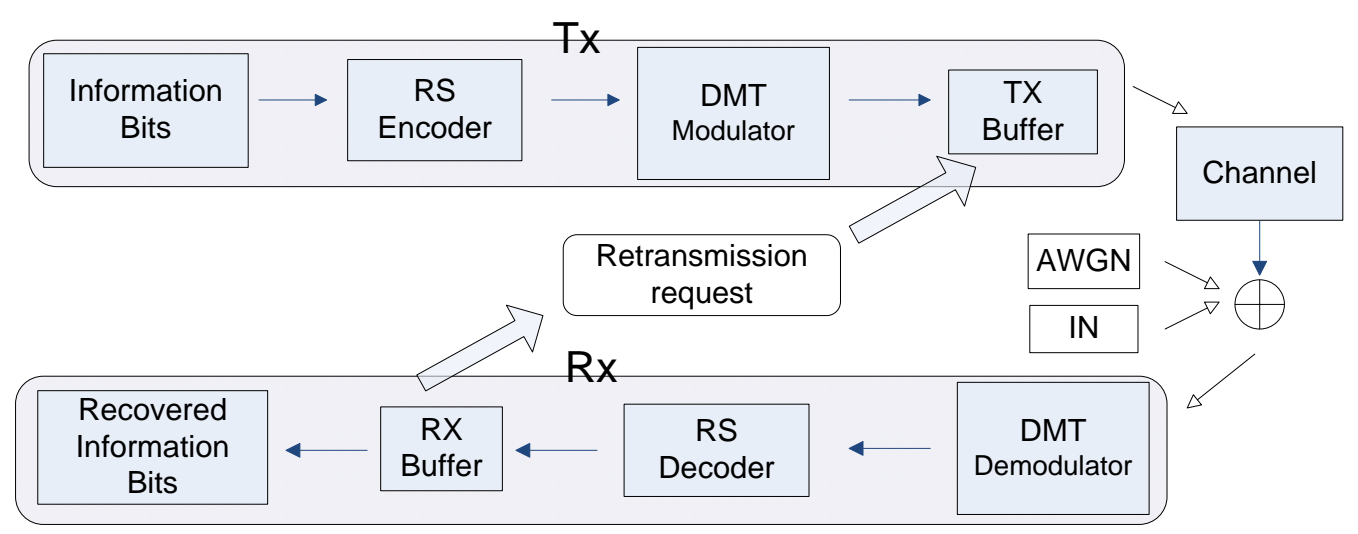

Figure 3.1. DSL system model employing retransmission techniques

Figure 3.1 shows the block diagram of a typical DSL system employing the retransmission technique. Compared with the existing DSL system in Figure 2.1, the interleaving component is removed from the system. Instead, the buffers are provided

\footnotetext{
${ }^{2}$ Part of this chapter has been presented in [32].
} 
both at the transceiver and receiver sides, allowing for data storage in the buffer before the system acknowledges a successful reception.

At the transmitter, information bit sequence is firstly RS-encoded to generate coded bits which are then DMT-modulated. The resulting DMT symbols are passed through the channel and corrupted by both IN and AWGN. A transmit (Tx) buffer is used to store transmitted DMT symbols, to resend upon the request from the receiver. The transmitter sends new DMT symbols continuously until it receives retransmission request (NAK) from the receiver. Then the requested DMT symbol is taken out of the Tx buffer and transmitted through the channel. The received sample $R_{k}$ at the output of FFT is demodulated (i.e., mapped to the nearest $M_{k}$-QAM symbol) and then the resulting data is passed to the RS decoder to recover the transmitted information. The recovered information is not passed to the upper layer right away but stored in the receive $(\mathrm{Rx})$ buffer for further checking as shown in Figure 3.1. If a recovered DMT symbol is detected as being in error, the receiver will send a retransmission request for this DMT symbol to the transmitter through the feedback channel which is assumed to be error-free in this study ${ }^{3}$. It is obvious that in order to implement the retransmission technique in DSL systems, buffers are required at the physical layer in both the transmitter and receiver to store copies of DMT symbols.

In the system model, selected buffer size limits the maximum allowable number of retransmissions. For example, assuming that the DMT symbol (say, T1) is unsuccessfully decoded, the retransmission request (NAK1) for T1 is sent back to the transmitter. In this case, even though the received DMT symbol (R11) corresponding to $\mathrm{T} 1$ is in error, it is still stored in the receive buffer. After receiving NAK1, a copy of T1 is not resent immediately since the other DMT symbol (say, T2) is being transmitted (see Figure 3.2). As a result, the transmitter waits until the transmission of $\mathrm{T} 2$ is completed and then retransmits T1, which will then be received at the receiver as R12. The receiver again determines whether it is necessary to request another retransmission

\footnotetext{
${ }^{3}$ The feedback channel is assumed to be error-free, i.e., retransmission requests (NAKs) are never lost when the receiver requests the retransmission.
} 
for $\mathrm{T} 1$, and stores R12 into the receive buffer. The first coming symbols in the receive buffer will be rejected if the buffer is full.

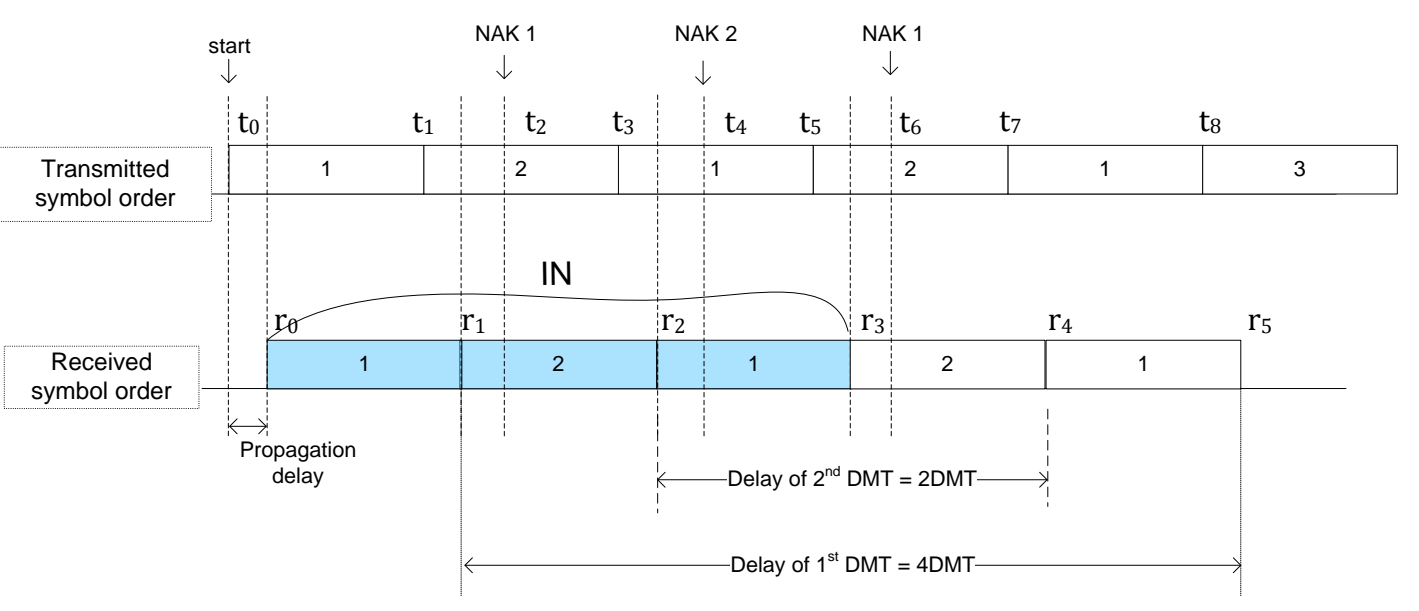

Figure 3.2. Illustration of retransmission process

For each retransmission requested, the fast retransmission only causes a latency length of two DMT symbol plus a propagation delay. So the additional delay produced by retransmission is calculated by the number of retransmissions (denoted as $v-1$ for a DMT symbol which is transmitted $v$ times) with a retransmission time of $T=$ $2 T_{D M T}+T_{p}$ where $T_{D M T}$ is the duration of one DMT symbol (i.e., $250 \mu \mathrm{s}$ in xDSL) and $T_{p}$ is the propagation delay (e.g., $2.5 \mu$ s for a DSL cable length of $500 \mathrm{~m}$ ). Let $P_{v}$ be the probability of the $v$ (re)transmission times for a DMT symbol. The average additional delay due to retransmission is given as:

$$
T_{a v g}=\sum_{v=1}^{v_{\max }}(v-1) T P_{v}
$$

where $v_{\max }$ is the maximum allowable number of retransmissions.

To limit the system delay, one can define the size of the buffer. An example is shown in Figure 3.3, where the buffer size is four DMT symbols, and the first two DMT symbols assumed to be impaired by IN have to be retransmitted. Whenever a new DMT symbol is sent, it is also stored in the Tx buffer. As well, all the received DMT symbols including the retransmitted ones are saved in the Rx buffer. With the buffer size of four 
DMT symbols, the receiver knows that buffering the first DMT symbol three times is impossible. As a result, the receiver does not request the two-time retransmission for it (i.e., NAK1 will not be issued even though the second reception of the first symbol is unsuccessful). Consequently, the third DMT symbol instead of the first DMT symbol is transmitted and the receiver accepts erroneous reception of the first DMT symbol. As seen in Figure 3.3, each symbol can be retransmitted once for the buffer size of four DMT symbols. So we can derive the equation for $v_{\max }$, which is limited by the buffer size of the receiver $N_{B u f f e r}$ :

$$
v_{\max }=\left\lceil\frac{N_{B u f f e r}}{2}\right\rceil,
$$

where $\lceil$.$\rceil is the ceiling opterator.$

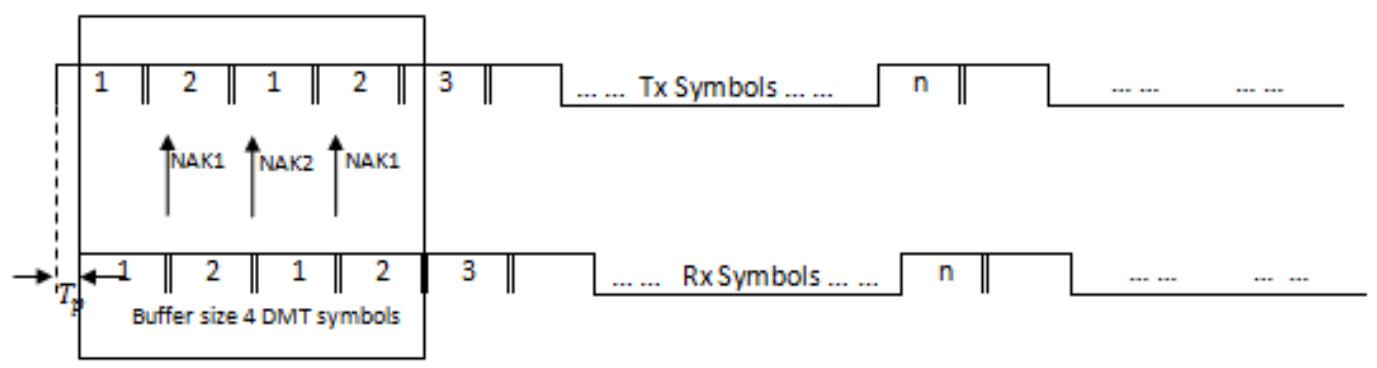

\section{Figure 3.3. Example of retransmission scheme with buffer size of 4 DMT symbols}

\subsection{Retransmission Criteria}

As discussed before, it is critical to determine when to retransmit a symbol in the implementation of a retransmission technique. In this thesis, we consider three criteria for retransmission.

\subsubsection{Criterion A- Decoding Status}

We propose to use the available RS decoding failure status to trigger the retransmission request as follows. The RS code allows the receiver to perform both error detection and correction. For example, the RS decoder directly corrects the 
erroneous bytes in one codeword after decoding. If the RS decoder cannot recover the codeword, it also reports the failure of decoding.

Assume that, the RS encoder takes $k$ bytes data and adds $(n-k)$ parity bytes to make an $n$-byte codeword. The algorithm of $\mathrm{RS}$ encoding requires an algebraic operation over a finite field known as Galois Fields $(G F)$. Define $\mathrm{GF}(q)$ as a finite GF field that contains $q$ elements. The RS code makes use of GF with $q=2^{m}$, where elements of the field can be represented by $m$ bits.

In this work, we apply an $\left(N_{R S}, K_{R S}\right) \mathrm{RS}$ code, which is shortened from $(n, k) \mathrm{RS}$ code. Note that these two codes are in the same $\operatorname{GF}(q)$. For instance, $(255,239)$ RS code and its shortened $(128,112) \mathrm{RS}$ code are both in $\mathrm{GF}(256)$ with the symbol size of $m=8$ bits. The error correction capability of the $\left(N_{R S}, K_{R S}\right)$ RS code is $t=\left\lfloor\frac{d_{\min }-1}{2}\right\rfloor$, where $\lfloor\cdot\rfloor$ denotes the floor operator and $d_{\min }=N_{R S}-K_{R S}+1$ is the minimum distance. Once the number of errors exceeds the error correction capability, there are two other possibilities: either the decoder fails to find any valid codeword, which indicates decoding failure, or it finds a valid codeword different from the transmitted one, causing decoding error [26, 27]. In the case of decoding failure, this decoding status can be used to trigger a retransmission request when the decoder fails to recover the transmitted information. In the sequel, we refer to "Decoding Status" as Criterion A.

As data is transmitted in units of DMT symbols where each DMT symbol contains several RS codewords (e.g, $Q$ RS codewords per DMT symbol), the retransmission request for a whole DMT symbol is issued when one or more RS codewords in this DMT symbol are detected as decoding failure. Therefore, a necessary error detection is missed (and hence retransmission is not made) only when, in this DMT symbol, there are one or more decoded codewords but no decoded codewords indicating decoding failure. In this case, the DMT symbol is wrongly decoded.

Besides the use of available decoding failure status, the presence of IN can be investigated to trigger the retransmission request. We propose the following criteria to detect the presence of IN. 


\subsubsection{Criterion B - Erasure Marking}

The "Erasure Marking" criterion marks the received unreliable symbols as erasures before decoding. A demodulated symbol is considered to be unreliable if the Euclidean distance between it and its received sample exceeds a selected threshold [17].

Figure 3.4 shows possible original positions for the modulated/demodulated QAM symbols, where each point represents one position. Let $d$ denote the distance between

two adjacent modulated symbols and $R$ the distance threshold. Also, let $\gamma=R /\left(\frac{d}{2}\right)$ be a ratio threshold. The term $d^{2}$ is proportional to the power of the transmitted signal. The area circled by radius $R$ can be setup as proportional to the tolerable noise range. In Figure 3.4, the noise during the transmission moves the modulated symbol a certain distance away from its corresponding position. The received sample $R_{k}$ is expected to be inside the circular area of the corresponding area of transmitted $A_{k}$ when it is only affected by background AWGN. However, if IN affects the symbol, the received symbol $R_{k}$ could be outside the circular area; and thus, it will be marked as one suspected erasure. It is important to note that the impact of IN with high amplitude causes a relatively large deviation in distance. To mark erasures efficiently and reliably, we should optimize the threshold $\gamma$ in such a way that IN-impaired symbols are marked with a high accuracy while AWGN affected symbols should not be marked. 


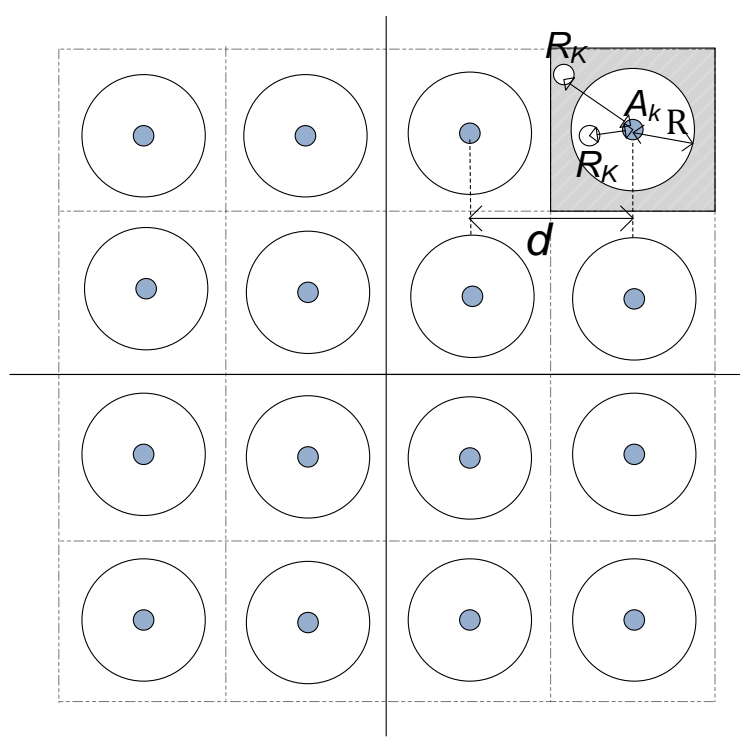

\section{Figure 3.4. Square-distance-based erasure marking}

There are two fallacious events related to this optimization problem. Firstly, although IN is present, it may happen that IN is not strong enough and the received sample might lie inside the circle area. Or it is too strong that the received sample falls into another circle area. Thus, the sample should be erased, but it is not. This event is called missdetection [11]. The miss-detection probability is denoted by $P_{m}$. Secondly, when IN is not present, there is some chance that the received $R_{k}$ lies outside the circular area. Hence, the unnecessary erasure is marked for this sample, resulting in a false alarm [11]. The probability of a false alarm is denoted as $P_{f}$. It is noteworthy that neither fallacious event is expected to happen frequently. We will solve for an optimum threshold for minimizing $P_{f}+P_{m}$ later.

After the optimum $\gamma$ has been obtained, for each partition in the DMT symbol, we count the number of erasures. Define threshold $\beta$ as the ratio of the maximum allowed erasure bytes in one codeword to the RS-codeword length $N_{R S}$. If $\beta$ is too large, retransmission is requested only when many samples are marked as erasures. This may result in missing some necessary retransmission requests. If $\beta$ is too low, the system can be too sensitive and generate more unnecessary retransmissions. It is known that with 
the erasure marking procedure, RS code can provide an error correction capability of $\left(N_{R S}-K_{R S}\right)$ [11]. In this case, a proper guess is to let $\beta=\left(N_{R S}-K_{R S}\right) / N_{R S}$.

We propose two types of comparison to track the unreliably received samples. The first one, defined as Criterion B-1, is to check every RS codeword in a DMT symbol. If the number of erased bytes exceeds a certain proportion in either one of the $Q$ RS codewords, the retransmission for the whole DMT symbol is requested. Nevertheless, since the retransmission is requested for each DMT symbol, the procedure for counting the number of erasures can be taken once per DMT symbol. The second case, defined as Criterion B-2, is to count all the erased samples in the DMT symbol. If the total number is larger than a scheduled number, the symbol must be retransmitted. Compared with B-2, Criterion B-1 addresses the retransmission more similarly to Criterion A, where the checking procedure is taken within each codeword.

\subsubsection{Criteron C - 'Unused' Tone Status}

In DSL systems, some tones in the allocated frequency band do not carry information due to high channel attenuation. For instance, in DSL profile-12a [18], for downstream transmission, only tones in DS1 and DS2 bands are used to carry data. One example for the bit loading in one DMT symbol is shown in Figure 3.5, where tones in DS3 band are considered as unused (i.e., the transmit power is zero on those tones). 


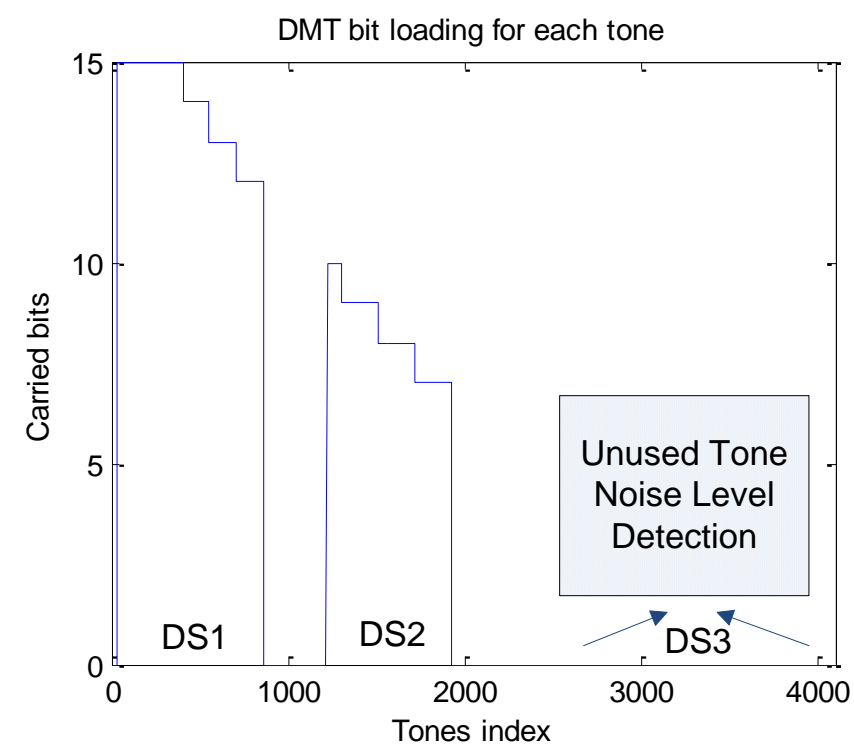

Figure 3.5. System model with retransmission and unused-tone-based IN detection

The received signals $R_{k}$ on unused tones can be inferred from (2.5) without the first term. On these tones, we have two hypotheses: the first hypothesis $\left(H_{0}\right)$ corresponds to the event of the absence of IN and the second one $\left(H_{1}\right)$ corresponds to the event of the presence of IN:

$$
\begin{gathered}
H_{0}: R_{k}=W_{k}, \\
H_{1}: R_{k}=I_{k}+W_{k},
\end{gathered}
$$

where $k \in \mathrm{U}$ with $\mathrm{U}$ being a set of unused tone indices with the cardinality of $N_{u}$.

It can be seen that

$$
\frac{1}{N_{u}} \sum_{k \in U}\left|R_{k \mid H_{0}}\right|^{2}<\frac{1}{N_{u}} \sum_{k \in U}\left|R_{k \mid H_{1}}\right|^{2},
$$

where $R_{k \mid H_{1}}$ and $R_{k \mid H_{0}}$ stand for the received signals on the tone $k$ with and without IN (corresponding to $H_{1}$ and $H_{0}$ ), respectively. The left hand side of (3.3) represents the average noise power in the unused frequency band without the presence of IN (i.e., AWGN only), whereas the right hand side represents the average noise power on empty 
tones in the presence of IN. When $N_{u}$ is large, according to the law of large numbers, the above inequality becomes

$$
E\left\{\left|R_{k \mid H_{0}}\right|^{2}\right\}<E\left\{\left|R_{k \mid H_{1}}\right|\right\}^{2}
$$

that is

$$
\sigma_{G}^{2}<\sigma_{G}^{2}+\sigma_{I}^{2}
$$

where $E\{$.$\} denotes the expectation and \sigma_{I}^{2}$ is the average impulse noise power.

From (3.5), by observing the noise power in the unused frequency band, the presence of IN can be detected and the retransmission request can be issued. Instead of GLRT for IN detection presented in [10], this thesis considers the following simpler IN detector. If the noise power measured from unused tones exceeds a certain threshold $\alpha$ (i.e., $\sum_{k \in U}\left|R_{k}\right|^{2}>\alpha=\sigma_{G}^{2}+\gamma_{u}$ ), the presence of IN is declared. The term $\sigma_{G}^{2}$ is the average noise power measured from the unused tones which are not affected by IN and can be determined off-line. DSL systems still work properly when disturbances other than AWGN fall within the target SNR margin $\gamma_{m}$. Therefore, it is not necessary to ask for retransmission when the average impulse noise power $\sigma_{I}^{2}$ in (3.5) is under SNR margin (i.e., $\left.\sigma_{I}^{2} \leq \gamma_{m}\right)$. The value of $\gamma_{u}$ represents the trade-off between the BER and delay. The lower $\gamma_{u}$, the higher delay since retransmission will occur more frequently. However, the bit error rate can be reduced. That is because the transmitted signals are rarely passed through the buffer when the low power impulse noise corrupts the transmission. On the other hand, the higher $\gamma_{u}$, the lower delay and the higher probability that BER would be large.

To determine whether one DMT symbol needs to be retransmitted, the received signal power on the empty tones is calculated. If this power exceeds the threshold $\alpha$, the retransmission request is sent back to the transmitter. Otherwise, the recovered information is passed to the upper layer and errors may happen, depending on the selected threshold. We define this retransmission criterion as C-1. 
Instead of the observing the power of noise over the whole 'unused' band, we propose Criterion C-2 as follows. We check the number of subcarriers on which the noise power exceeds $\alpha$. If this number is higher than $N_{u}$, the DMT symbol is retransmitted. We expect a more precise detection of IN since the observation is done with each tone, and this decision can be more flexibly made with the modification of $N_{u}$.

\subsection{Performance Analysis}

In the previous section, we introduce three different criteria for the requirement of retransmission. To check whether each criterion efficiently tracks the unreliable symbols, there are several factors that can be evaluated analytically. In this section, we will derive the probability of retransmission times, the average delay, and the BER with and without retransmission. For convenience, we use Bernoulli-Gaussian IN in the analysis.

\subsubsection{BER Derivations}

BER is one important figure of merit to evaluate the system performance. In this section, we focus on the derivation of BER for the system without and with retransmission procedure.

At first, the symbol error rate for each $M_{k}$-QAM symbol is [24]

$$
P_{s_{l, k} \mid M_{I N}}=4 K_{k} Q\left(\sqrt{2 g_{Q A M, k} \gamma_{k \mid M_{I N}}}\right)-4 K_{k}^{2} Q^{2}\left(\sqrt{2 g_{Q A M, k} \gamma_{k \mid M_{I N}}}\right)
$$

where $\gamma_{k \mid M_{I N}}=\frac{\left|C_{k}\right|^{2} E\left\{\left|A_{k}\right|^{2}\right\}}{\sigma_{G, M_{I N}}^{2}}$ represents the SNR on each tone; $\sigma_{G, M_{I N}}^{2}=\sigma_{G}^{2}+\sigma_{I, M_{I N}}^{2}$ is the total noise power with $\sigma_{I, M_{I N}}^{2}=\frac{M_{I N}}{N} \sigma_{i}^{2}$, and $M_{I N}$ is the number of the time-domain IN samples occurring in the DMT symbol duration under consideration; $K_{k}=1-\frac{1}{\sqrt{M_{k}}}$; $g_{Q A M, k}=\frac{3}{2\left(M_{k}-1\right)} ; M_{k}$ is the QAM size on the $k^{t h}$ tone; $s_{l, k}$ represents the $k^{t h}$ symbol in the $l^{\text {th }}$ codeword in the DMT symbol. 
The probability that the $u^{\text {th }}$ byte of the $l^{\text {th }} \mathrm{RS}$-codeword in the DMT symbol, $B_{l, u}$, is in error, conditioned on $M_{I N}$ is given by

$$
\begin{aligned}
P_{B_{l, u} \mid M_{I N}} & =1-\prod_{k \in B_{l, u}}\left(1-\frac{P_{s_{l, k} \mid M_{I N}}}{\log _{2} M_{k}}\right)^{h_{k}} \\
& \approx \sum_{k \text { in } B_{l, u}} \frac{h_{k} P_{s_{l, k} \mid M_{I N}}}{\log _{2} M_{k}}
\end{aligned}
$$

where $h_{k}$ is the number of bits of $B_{l, u}$ carried by tone $k$ carries (i.e., belonging to $M$ QAM symbol $\left.s_{l, k}\right)$. The probability of byte error conditioned on $M_{I N}, P_{B \mid M_{i, I N}}$, is derived by taking the average of $P_{B_{l, k} \mid M_{I N}}$ over all the bytes $u$ and RS-codewords $l$ in the DMT symbol. As shown in (3.7), for a frequency-selective DSL channel, different tones can use different $M_{k}$-QAM sizes depending on their SNR's and hence can have different symbol error rates $P_{s_{l, k} \mid M_{I N}}$. As a result, averaging $P_{B_{l, u} \mid M_{I N}}$ over all the bytes $u$ and RS-codewords $l$ is complicated. In that case, the derived equation for the whole transmission would be hard to deduce. An example is shown in Figure 3.6, where we assume Gray-coded modulation and independent bits ${ }^{4}$. Each top block in Figure 3.6 describes the number of bits carried in $k^{\text {th }}$ tone and the block below represents the $u^{\text {th }}$ byte transmitted. For example, the $1^{\text {st }}$ tone carrying 15 bits (i.e., modulation size $2^{15}$ QAM) has the probability of bit error of $\frac{P_{S_{1}}}{\log _{2} 15}$. Similarly, $\frac{P_{S_{2}}}{\log _{2} 13}$ is the probability of bit error for the $2^{\text {nd }}$ tone carrying 13 bits. The first byte in this DMT symbol is only contained in the $1^{\text {st }}$ tone so that its byte error rate is $P_{B_{l, 1}}=1-\left(1-\frac{P_{s_{l, 1}}}{\log _{2} 15}\right)^{8}$. However, the second byte related to the first two symbols, has the byte error rate of $P_{B_{l, 2}}=1-\left(1-\frac{P_{s_{l, 1}}}{\log _{2} 15}\right)^{7}\left(1-\frac{P_{s_{l, 2}}}{\log _{2} 13}\right)$. To obtain further calculations, byte error rate with (3.7) can lead to very complicated equations.

\footnotetext{
${ }^{4}$ The independence between the bits may not be valid without the bit interleaver
} 


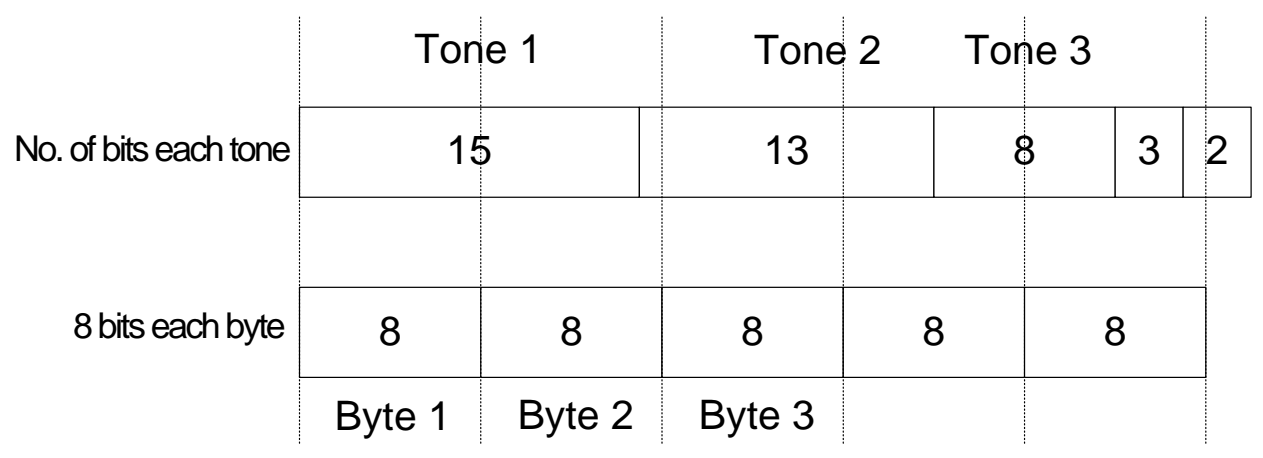

\section{Figure 3.6. Codeword error rate calculation with frequency selective fading channel}

To simplify the analysis and to get an insight on the impact of IN and retransmission technique, as an approximation, we can consider the average of the tone SNR's, and use it to determine the corresponding $M_{k}$-QAM size $M_{k}$ and symbol error rate $P_{S \mid M_{I N}}$ so that, from (3.9), the probability of byte error conditioned on $M_{I N}$ becomes

$$
P_{B \mid M_{I N}} \approx \frac{8 P_{S \mid M_{I N}}}{\log _{2} M_{k}}
$$

This is equivalent to the case of a frequency-flat channel response.

Since the RS decoding fails when the number of errors exceeds the error correction capacity, assuming that the byte errors are independent, the average number of erroneous bytes $N_{B}$ after decoding in each codeword per DMT symbol conditioned on $M_{I N}$ can be calculated as

$$
N_{B \mid M_{I N}}=\sum_{s=t+1}^{N_{R S}} s\left(\begin{array}{c}
N_{R S} \\
s
\end{array}\right) P_{B \mid M_{I N}}^{S}\left(1-P_{B \mid M_{I N}}\right)^{N_{R S}-s} .
$$

where $N_{R S}$ is the number of bytes per codeword, $s$ is the possible number of erroneous bytes in each codeword.

The conditional probability of byte error for the DMT after decoding is 


$$
P_{B a f t \mid M_{I N}}=\frac{P_{B \mid M_{I N}}}{N_{R S}}
$$

From the conditional probability of byte error, $P_{B a f t \mid M_{I N}}$, we can approximately calculate the conditional probability of bit error of the DMT after decoding as

$$
P_{\text {baft } \mid M_{I N}} \approx \frac{P_{B a f t \mid M_{I N}}}{8}
$$

Considering the cases of different number of IN samples contaminating the DMT symbol, the average probability of bit error in the DMT after decoding is

$$
P_{b a f t}=\sum_{M_{I N=0}}^{N} P_{M_{I N}} P_{\text {baft } \mid M_{I N}}
$$

The bit error rate (BER) after $v^{\text {th }}($ re)transmission can be derived from (3.10) and the probabilities of retransmission times for different criteria as shown in the following sections.

\subsubsection{Performance Analysis for Criterion A - Decoding Status}

When a codeword $c_{i}$ is transmitted over a communication channel, channel noise corrupts the codeword so that $c_{i}+e_{i}$ is received, where $e_{i}$ is an error pattern of some weight $u$. We have three possibilities for the status of the received codeword [26-28]:

(i) Successfully decoding with probability $P_{c_{i} \mid M_{I N}}$. This happens when $u \leq t$, i.e., the decoder detects and corrects $e_{i}$ and recovers $c_{i}$.

(ii) The decoder detects the presence of the error pattern. However, it confirms that it is unable to correct it (i.e., decoding failure with probability $P_{F_{i} \mid M_{I N}}$ ).

(iii) The decoder miscorrects $c_{i}$ into $c_{i}$ ' if the received $c_{i}+e_{i}$ falls into the radius $t$ Hamming sphere of $c_{i}{ }^{\prime}$ (i.e., decoding error with $P_{E_{i} \mid M_{I N}}$ ).

It follows that 


$$
P_{F_{i} \mid M_{I N}}=1-P_{c_{i} \mid M_{I N}}-P_{E_{i} \mid M_{I N}} .
$$

Since the byte error rate is simplified in (3.10), we can find the probability that the codeword is successfully decoded as

$$
P_{c_{i} \mid M_{I N}}=\sum_{S=1}^{t}\left(\begin{array}{c}
N_{R S} \\
s
\end{array}\right) P_{B_{i} \mid M_{I N}}^{S}\left(1-P_{B_{i} \mid M_{I N}}\right)^{N_{R S}-s} .
$$

The probabilities of case (ii) decoding failure and (iii) decoding error will be derived next.

Consider an $\left(N_{R S}, K_{R S}\right)$ code is shortened from an original $(n, k)$ RS-code defined over the $\operatorname{GF}(q)$ (i.e., $N_{R S} \leq n, K_{R S} \leq k$ and $N_{R S}-K_{R S}=n-k$ ). The study in [26] introduces the geometric interpretation of error correction - a Hamming sphere of radius $t$ that contains all the possible received vectors at Hamming distance less than $t$ from a codeword. A random error pattern could cause decoder error, which lies within distance $t$ of a codeword. The term $V_{n}(t)$ is defined as the volume of a Hamming sphere with radius $t$ in $n$-dimensional vector space over $\mathrm{GF}(q)$, where

$$
V_{n}(t)=\sum_{i=0}^{t}\left(\begin{array}{l}
n \\
i
\end{array}\right)(q-1)^{i} .
$$

A decoding error occurs when an error pattern has weight $u \geq d_{\min }-t$, and falls into the decodable area of another codeword [28]. The term $P_{E}(u)$ is used to denote the decoder error probability given that an error pattern of weight $u$ occurs. As shown in [27] establishes that $P_{E}(u)$ approaches the ratio of the number of decodable words over the cardinality of the whole vector space $Q_{v}$, where

$$
Q_{v}=\frac{\left(q^{k}-1\right) V_{n}(t)}{q^{n}} .
$$

For a more precise solution than the approximation in (3.14), the author in [28] derives the error patterns for different weights and assumes that all error patterns of weight $u$ are equiprobable. Let $D_{u}$ denote the number of decodable words (i.e., words 
within distance $t$ from a valid codeword) of weight $u$. A simplified weight enumerator was derived in [28] to compute $D_{u}$ as follows:

$$
D_{u}=\left(\begin{array}{l}
n \\
u
\end{array}\right) \sum_{j=0}^{u-d_{\min }+t}(-1)^{j} N_{j}, \quad \text { for } d_{\min }-t \leq u \leq n
$$

where $N_{j}$ is the number of objects with property $P(j)$ from $\mathrm{GF}(q)$ :

$$
\begin{aligned}
& N_{j} \\
& =\left\{\begin{array}{c}
\left(\begin{array}{l}
n \\
j
\end{array}\right)\left[q^{u-d_{\min }+1-j} V_{n}(t)-\sum_{i=0}^{t}\left(\begin{array}{c}
u-j \\
i
\end{array}\right)(q-1)^{i}\right] ; \text { for } 0 \leq j \leq u-d_{\text {min }} \\
\left(\begin{array}{c}
n \\
j
\end{array}\right)\left[\begin{array}{c}
\sum_{w=d_{\min }-u+j}^{t}\left(\begin{array}{c}
n-u+j \\
w
\end{array}\right) \sum_{i=0}^{w-d+i-j}(-1)^{i}\left(\begin{array}{c}
w \\
j
\end{array}\right) * \ldots \\
\left(q^{w-d_{\min }+u-j-i+1}-1\right) \sum_{s=w}^{t}\left(\begin{array}{c}
u-j \\
s-w
\end{array}\right)(q-1)^{s-w}
\end{array}\right] ; \\
\text { for } u-d_{\min }+1 \leq j \leq u-d_{\min }+1
\end{array}\right.
\end{aligned}
$$

Then the conditional decoding error probability $P_{E}(u)$ is

$$
P_{E}(u)=\frac{D_{u}}{\left(\begin{array}{l}
n \\
u
\end{array}\right)(q-1)^{u}} . \quad \text { for } d_{\text {min }}-t \leq u \leq n
$$

Let $q_{u}$ denote the probability that the error pattern has weight $u$. The error decoding probability conditioned on $M_{I N}$ would be [27]

$$
P_{E_{i} \mid M_{i, I N}}=\sum_{u=d_{\min }-t}^{N_{R S}} P_{E}(u) q_{u \mid M_{I N}}
$$

where $q_{u \mid M_{I N}}=\left(\begin{array}{c}N_{R S} \\ u\end{array}\right) P_{B \mid M_{I N}}^{u}\left(1-P_{B \mid M_{I N}}\right)^{N_{R S}-u}$. It follows that the error decoding probability is 


$$
P_{E_{i}}=\sum_{M_{I N}} P_{E_{i} \mid M_{I N}} P_{M_{I N}}
$$

where $P_{M_{I N}}$ represents the probability that one DMT symbol would be affected by $M_{I N}$ time-domain IN samples:

$$
P_{M_{I N}}=\left(\begin{array}{c}
N \\
M_{I N}
\end{array}\right) p^{M_{I N}}(1-p)^{N-M_{I N}}
$$

As previously discussed in Section 3.2.1, the retransmission of one DMT symbol (with $Q$ codewords) is requested when the encoder fails to decode either one codeword. Since retransmission is invoked whenever there is one or more decoding failures, the probability of retransmission $P_{r \mid M_{I N}}$ can be expressed as

$$
P_{r \mid M_{I N}}=1-\left(1-P_{F_{i} \mid M_{I N}}\right)^{Q} .
$$

Also as discussed in Section 3.2.1, there is some chance that the DMT symbol is wrongly decoded. This happens when a DMT symbol contains no decoded codeword with decoding failure but has $q_{e}>0$ codewords wrongly decoded. In other words, the probability that the DMT symbol is wrongly decoded can be presented as

$$
P_{E_{D M T}}=\sum_{M_{I N}}\left(\sum_{q_{e}=1}^{Q}\left(\begin{array}{c}
Q \\
q_{e}
\end{array}\right) P_{E_{i} \mid M_{I N}}^{q_{e}} P_{c_{i} \mid M_{i N}}^{Q-q_{e}}\right) P_{M_{I N}} .
$$

We can calculate the probability that one DMT symbol does or does not need retransmission, respectively, as: 


$$
\begin{gathered}
P_{R E T}=\sum_{M_{I N}=0}^{N} P_{M_{I N}} P_{r \mid M_{I N}} \\
P_{N R}=\sum_{M_{I N}=0}^{N} P_{M_{I N}}\left(1-P_{r \mid M_{I N}}\right) .
\end{gathered}
$$

Combining (3.22) and (3.23), the probability that one DMT symbol requires $v$ (re)transmissions is given by

$$
P_{v}=P_{R E T}^{v-1} P_{N R} .
$$

Using (3.1) with $P_{v}$ given by (3.24), we can calculate the average delay caused by retransmission using Criterion A.

\subsubsection{Performance Analysis for Criterion B - Erasure Marking}

For Criterion B, the retransmission is based on the number of erased samples. Firstly we are looking for the optimum threshold to precede the erasure marking. The probability of marking (i.e., samples fall into filled area in Figure 3.4) one DMT symbol affected by $M_{I N}$ samples of IN is

$$
P_{E r a \mid M_{I N}}=\left(1-P_{B \mid M_{I N}}\right)-\left(1-e^{-\frac{R^{2}}{\sigma_{G, M_{I N}}^{2}}}\right),
$$

As described in Section 3.2.2, when a received symbol lies outside the circular area (see Figure 3.4), it is marked as one suspected erasure. Equation (3.25) consists of two terms: the first term represents the percentage of symbols that fall into their own corresponding square area and the second term is the percentage of symbols that are inside their corresponding circular area. The filled area in Figure 3.4 shows one example of (3.25) inside which the symbol should be marked as erasure.

Erasure marking is more precise when the probability of miss-detected and falsedetected marked erasure is lower. In that sense, we aim to minimize the probability of $P_{m}+P_{f}$. Since the event of missed-detection is complementary to the event of good 
detection (where $P_{\text {cor }}$ is the probability that the received signal is erased correctly), i.e., $P_{m}=1-P_{c o r}$, we can maximize $P_{c o r}-P_{f}$ with respect to the choice of $R=\gamma\left(\frac{d}{2}\right)$. It can be shown that the probability of correct erasure is

$$
P_{c o r}=\sum_{M_{I N}=1}^{N} P_{M_{I N}}\left\{\left(1-P_{B \mid M_{I N}}\right)-\left(1-e^{-\frac{R^{2}}{\sigma_{G . M_{I N}}^{2}}}\right)\right\}
$$

This represents the probability that the $M_{I N} \geq 1$ samples of IN-contaminated DMT symbol are truly marked as erasure.

Then, the probability of false alarm is

$$
P_{f}=P_{M_{I N}=0}\left\{\left(1-P_{B \mid M_{I N}=0}\right)-\left(1-e^{-\frac{R^{2}}{\sigma_{G}^{2}}}\right)\right\}
$$

This represents the probability that even though the symbol is only affected by background noise, it is still marked as erasure.

With the solution to maximize $P_{\text {cor }}-P_{f}[$ see (3.26) and (3.27)], the optimum distance threshold $R$ can be found. With the fixed $R$, the retransmission of a $\mathrm{RS}$ codeword is required when the number of erased samples is larger than $N_{e}$. We denote this probability as $P_{r S \mid M_{I N}}$, where

$$
P_{r S \mid M_{I N}}=\sum_{s=N_{e}+1}^{n}\left(\begin{array}{c}
N_{R S} \\
s
\end{array}\right) P_{E r a \mid M_{I N}}\left(1-P_{E r a \mid M_{I N}}\right)^{N_{R S}-s}
$$

In the above equation, the threshold $N_{e}$ is defined to check the erased bytes in each RS codeword. As defined in Section 3.2.2, Criterion B-1 is that if the number of erased symbols exceeds a certain percentage $\beta=N_{e} / N_{R S}$ in one of those RS codewords, the whole DMT symbol retransmission is requested. Thus, the retransmission probability in this case is determined in the same way as that for Criterion A. For the whole DMT symbol, the retransmission probability is given by: 


$$
P_{r \mid M_{I N}}=1-\left(1-P_{r S \mid M_{I N}}\right)^{Q}
$$

The $v$-time retransmission probabilities are obtained by following (3.20) - (3.24) with $P_{r \mid M_{I N}}$ derived in (3.29) for Criterion B-1.

As for Criterion B-2, $\beta$ is defined as a proportion of the bytes erased in one DMT symbol. Let the total bytes in one DMT symbol be $B_{D M T}$. If the number of bytes erased in the DMT symbol exceeds $\beta B_{D M T}$, the DMT symbol is then retransmitted ${ }^{5}$.

\subsubsection{Performance Analysis for Criterion C -'Unused' Tone Status}

Criterion $\mathrm{C}$ is based on the noise power detected in the 'unused' tone. As discussed in the above, the retransmission is requested when the average noise power on the 'unused' tones is larger than $\alpha$, where $\alpha=\sigma_{G}^{2}+\gamma_{u}$ is the threshold:

$$
\frac{1}{N_{u}} \sum_{k=1}^{N_{u}}\left|R_{k}\right|^{2}>\alpha .
$$

Let $h=\sum_{k=1}^{N_{u}}\left|R_{k}\right|^{2}$, (3.30) becomes $h>N_{u} \alpha$. To find the probability of noise power outage, we look for the power density function (PDF) of $h$, which is a chi-square (or gamma) PDF with $2 N_{u}$ degrees of freedom:

$$
f_{H}(h)=\frac{1}{\sigma_{G, M}^{2 N_{u}} \Gamma\left(N_{u}\right)} h^{N_{u}-1} e^{-\frac{h}{\sigma_{G, M}^{2}}},
$$

where $\Gamma(\cdot)$ is the gamma function.

The probability to retransmit a DMT symbol is then:

\footnotetext{
${ }^{5}$ The equation for criterion B-2 is not provided due to the many possible combinations for the codewords in one DMT symbol to have a total number of error bytes of $\beta B_{D M T}$.
} 


$$
\begin{aligned}
P_{r \mid M_{I N}} & =\operatorname{Pr}\left\{\frac{1}{N_{u}} \sum_{k=1}^{N_{u}}\left|R_{k}\right|^{2}>\alpha \mid M_{I N}\right\} \\
& =\int_{N_{u} \alpha}^{\infty} \frac{1}{\sigma_{G, M_{i, I N}}^{2 N_{u}} \Gamma\left(N_{u}\right)} h^{N_{u}-1} e^{-\frac{h}{\sigma_{G, M_{i, I N}}^{2}}} d h \\
& =e^{-\frac{N_{u} \alpha}{\sigma_{G, M_{i, I N}}^{2}}} \sum_{k=0}^{N_{u}-1} \frac{1}{k !}\left(\frac{N_{u} \alpha}{\sigma_{G, M_{i, I N}}^{2}}\right)^{k}
\end{aligned}
$$

since $\Gamma\left(N_{u}\right)=\left(N_{u}-1\right)$ ! It is clear from the above equation that when the DSL transmission condition is fixed, the parameter affecting the retransmission probability is the threshold $\alpha$. Also, with $P_{r \mid M_{I N}}$ in (3.32), we can derive the probabilities of $v$-time retransmission by following (3.20) - (3.24) for Criterion C-1. 


\section{Chapter 4}

\section{Performance of Proposed Retransmission Schemes: Analytical and Simulation Results ${ }^{6}$}

The performance of the retransmission schemes proposed in Chapter 3 will be evaluated in this chapter by using both analysis (developed in Chapter 3) and simulation. Section 4.1 presents both analytical and simulation results of the proposed retransmission schemes in presence of Bernoulli-Gaussian IN for comparison. Section 4.2 provides further simulation results for DSL system with REIN and the frequency selective fading channel. Section 4.3 presents simulation results on performance of video transmission over DSL system with REIN and the frequency selective fading channel.

\subsection{Performance of proposed retransmission schemes in presence of Bernoulli-} Gaussian IN

The parameters under consideration are summarized in Table 4.1. The BernoulliGaussian IN is set up with an occupation $q=0.03$ and IN power spectral density in the range from $-105 \mathrm{dBm} / \mathrm{Hz}$ to $-30 \mathrm{dBm} / \mathrm{Hz}$. The low occupation rate of BernoulliGaussian IN ensures that in one DMT symbol transmission period, only one or two samples of Bernoulli-Gaussian IN would corrupt that symbol. The high level of IN ensures the affected DMT symbols have high probability to cause transmission error. For a frequency-flat channel response and 256-QAM, the probability of byte error conditioned on $M_{I N}$, is $P_{B \mid M_{I N}} \approx P_{S \mid M_{I N}}$.

\footnotetext{
${ }^{6}$ Part of this chapter has been presented in [32].
} 
Table 4.1. Analysis parameters

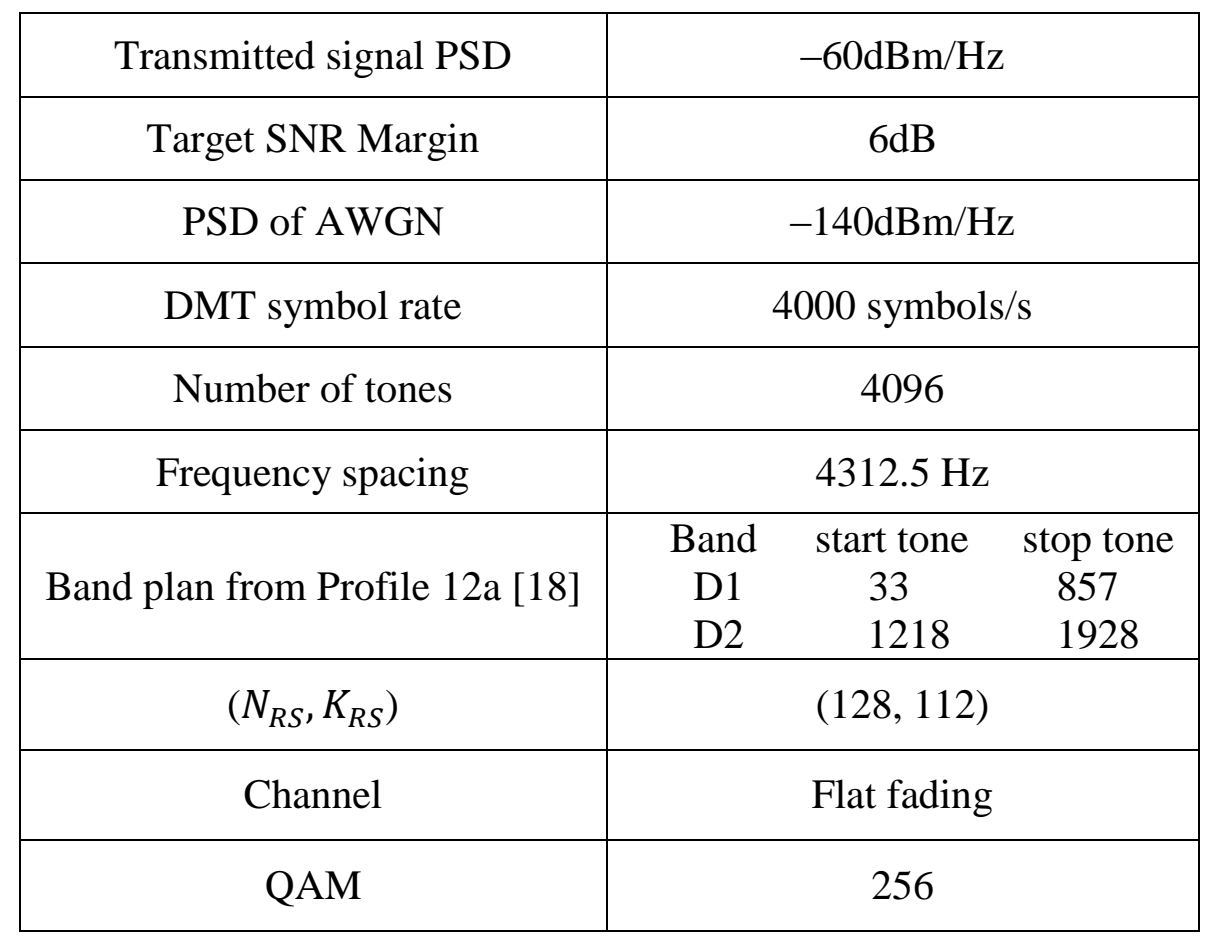

Besides the evaluation of BER and average retransmission delay, to see whether the retransmission request is reliable or not, one can observe DMT symbols impaired by IN versus the retransmission requests for them. One case is that the retransmission is required for some DMT symbol but IN does not affect that symbol; we call it false alarm for retransmission request. On the other hand, miss-detection means that IN corrupts a DMT symbol but the retransmission request is not issued. The false alarm probability is expected to be low because the chance of unsuccessful decoding is small without IN. The miss-detection probability is acceptable in a certain range since the INcontaminated symbol may have small number of errors that can be corrected by the RS decoder.

\subsubsection{Performance of Criterion A - Decoding Status}

We start the analysis with Criterion A, where the decoding status given by the RS decoder is the criterion to decide when to retransmit the symbol. To consider the 
"Decoding Status" as a reliable criterion, one must make sure that the wrong decoding probability for the DMT symbol is as low as possible.

Figure 4.1 shows the results on the decoding error probability $\left(P_{E_{i}}\right)$ and the probability of wrongly decoded DMT symbol $\left(P_{E_{D M T}}\right)$ versus IN PSD. Analytical results are obtained using (3.18) and (3.21) for $P_{E_{i}}$ and $P_{E_{D M T}}$, respectively. For the selected parameter values, the decoding error probability is around $5 \times 10^{-7}$ while the probability of wrongly decoded DMT symbol is lower than $2 \times 10^{-58}$ for PSD of IN higher than $-85 \mathrm{dBm} / \mathrm{Hz}$. For lower IN strength, this probability is dramatically reduced to near zero. Simulation results for $P_{E_{i}}$ are in good agreement with analytical results. As $P_{E_{D M T}}$ is very small, it is impossible to obtain simulation results for $P_{E_{D M T}}$ due to the requirement of impractically huge number of simulation events. The nearly zero value of $P_{E_{D M T}}$ for the entire range of IN PSD confirms the extremely high reliability of the proposed scheme using available RS decoding failure status in a DMT symbol to trigger the retransmission request.
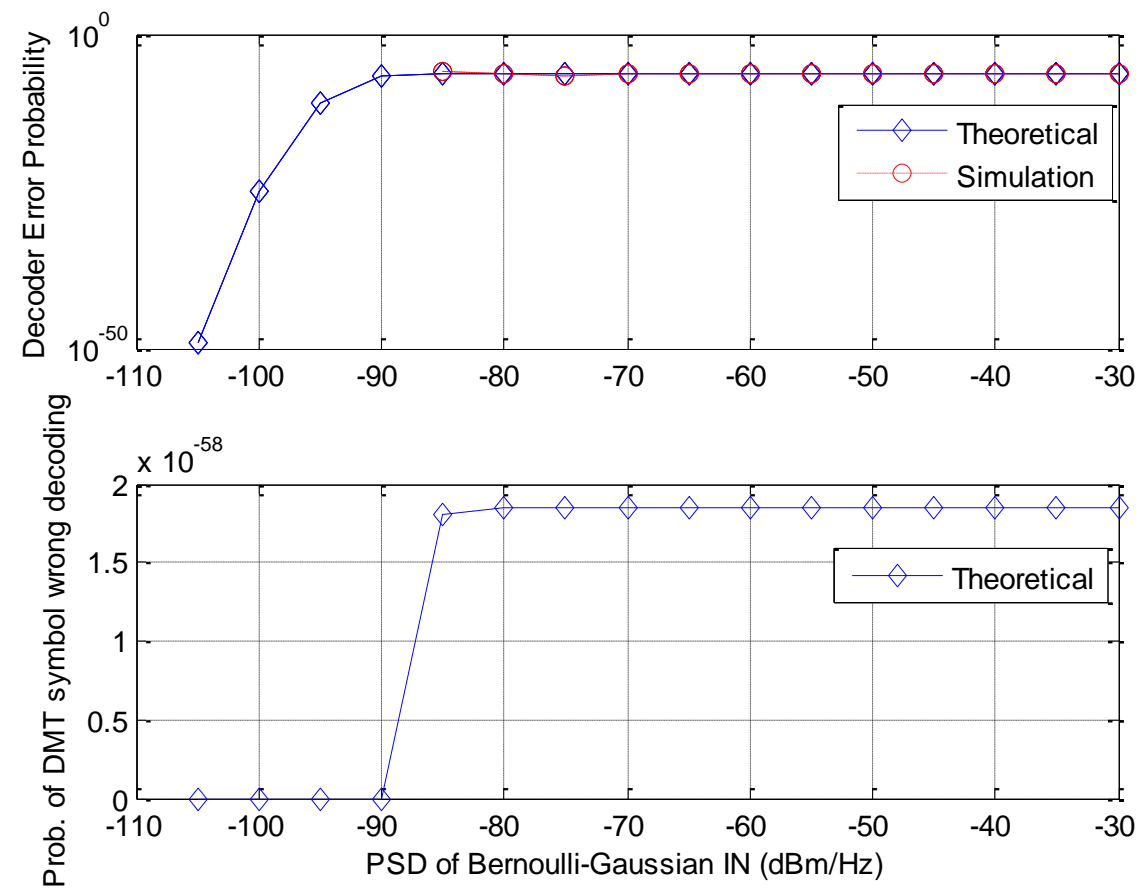

Figure 4.1. Probabilities of decoding error and DMT symbols being decoded wrongly 
At low IN occupation rate $(q=0.03)$, the average retransmission delay is mainly affected by the one-time retransmission possibility as shown in Figure 4.2. When IN is not strong with a PSD in the range from -105 to $-95 \mathrm{dBm} / \mathrm{Hz}$, RS decoder can correct most of the affected symbols; and hence, no retransmission is needed, corresponding to zero delay. When IN PSD increases to higher than $-90 \mathrm{dBm} / \mathrm{Hz}$, about $1.2 \%$ of DMT symbols require one-time retransmission, yielding an average delay of about $0.006 \mathrm{~ms}$. When IN PSD is about $-80 \mathrm{dBm} / \mathrm{Hz}$ or higher, around $2.8 \%$ of the DMT symbols are corrupted and require one-time retransmission. This corresponds to an average delay of $0.0145 \mathrm{~ms}$. Note that the amount $2.8 \%$ of DMT requiring retransmission is consistent with the IN occupation rate of $3 \%$.

Figure 4.2 indicates that with the same occupation rate of Bernoulli-Gaussian IN, the probability of one-time retransmission for a DMT symbol increases with the IN strength. Also, it remains the same when the PSD of IN is higher than $-80 \mathrm{dBm} / \mathrm{Hz}$. It can be concluded that Criterion $\mathrm{A}$ is able to offer an excellent performance.
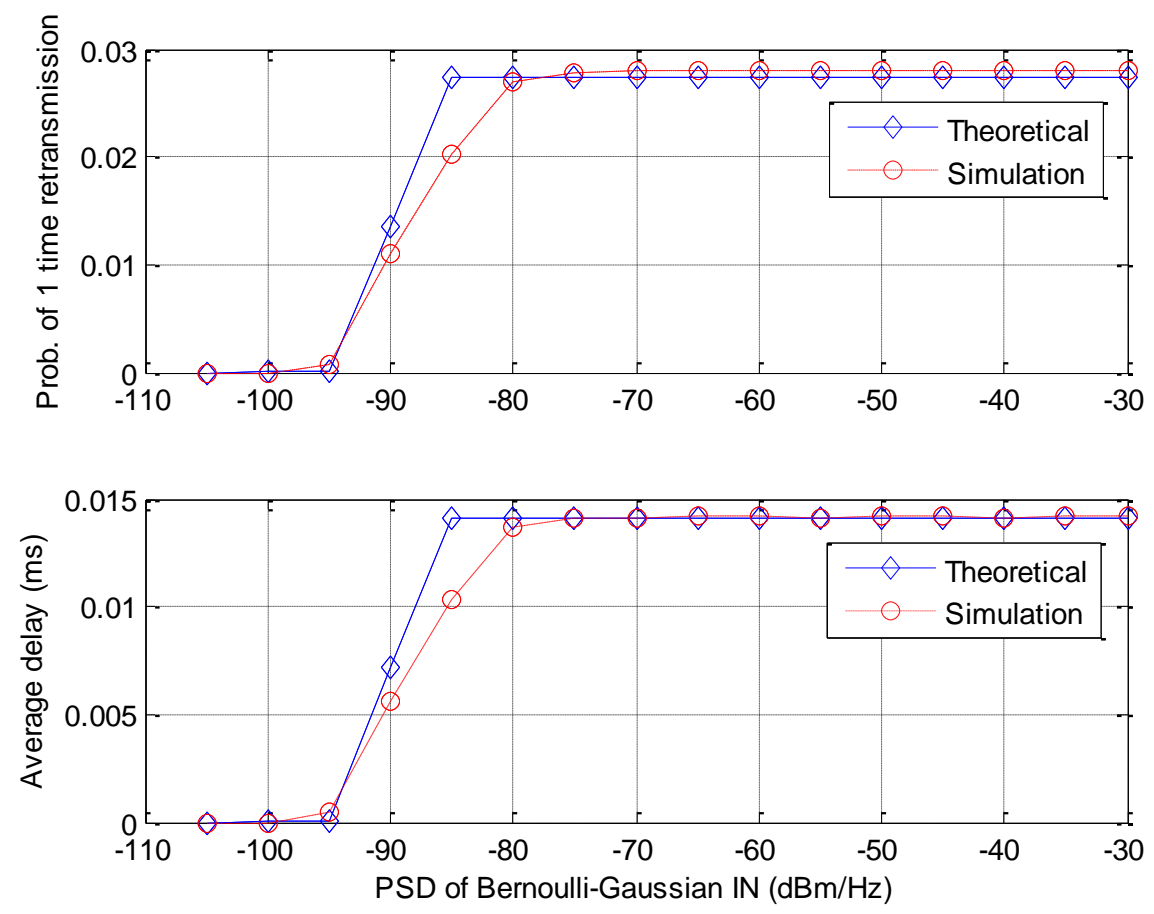

Figure 4.2. One-time retransmission probability $\left(P_{v}, v=2\right)$ and average delay $\left(T_{a v g}\right)$ versus IN PSD for Criterion A "Decoding Status" 


\subsubsection{Performance of Criterion B - Erasure Marking}

To ensure the performance of "Erasure Marking" criterion, one must optimize the threshold for erasure marking and retransmission request.

\section{(a) Square-distance threshold $(\gamma)$ optimization}

To perform a reliable erasure marking, the probability of miss-detection and false alarm during the observation of erasures for the received samples must be as small as possible. In this case, $\gamma$ is chosen to maximize $P_{c o r}-P_{f}$ (i.e., to minimize $P_{f}+P_{m}$ ) for IN with different power levels. The top plot in Figure 4.3 gives an example of how $\gamma$ affects the reliability of erasure marking, in which Bernoulli-Gaussian IN PSD is assumed $-90 \mathrm{dBm} / \mathrm{Hz}$. It can be observed that when $\gamma$ is equal to 0.5 , the maximum of $P_{c o r}-P_{f}$ is achieved.
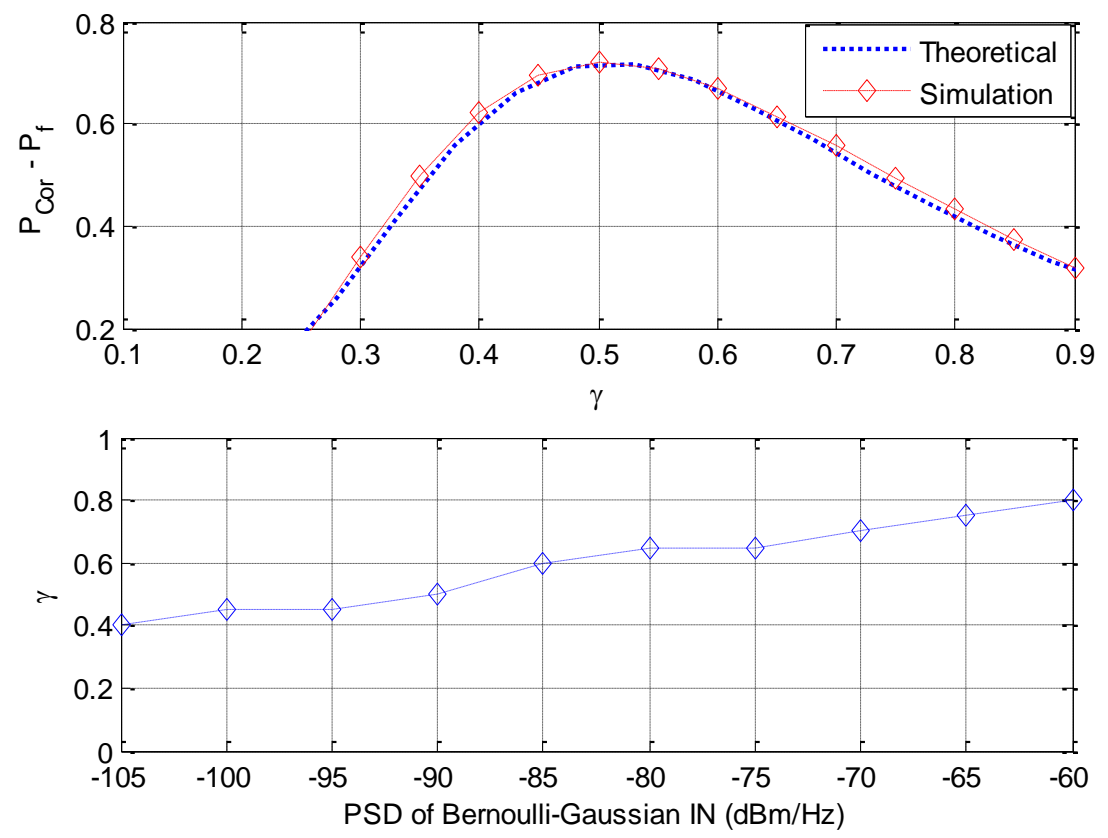

Figure 4.3. Example of square-distance threshold optimization and threshold selection versus IN PSD 
For the higher level of IN power, the symbol would be moved to a farther distance from its original position in the constellation. Here, we need to calculate and find out the optimized threshold for each case of IN power. The bottom plot in Figure 4.3 exhibits the choices of $\gamma$ for different strengths of Bernoulli-Gaussian IN. In general, $\gamma$ increases when IN is stronger.

\section{(b) Selection of threshold ( $\beta$ ) for number of marked erasures}

After optimizing $\gamma$ for each IN PSD, the other threshold $\beta$ will be selected. To choose $\beta$, we observe two results: (i) the probability of miss-detection for the retransmission, and (ii) the average retransmission delay variation. Here, we show one example with IN PSD of $-90 \mathrm{dBm} / \mathrm{Hz}$. The trends of the miss-detection probability $P_{M-D}$ and average retransmission delay versus threshold $\beta$ are shown in Figures 4.4 and 4.5 for criteria B-

1 and B-2, respectively. We test $N_{e}$ between 1 and $N_{R S}$ (i.e., $N_{e}=\beta N_{R S}$, or, $\frac{1}{N_{R S}} \leq \beta \leq$ 1).

In both figures, it can be seen that the increase of threshold $\beta$ causes the higher missdetection probability for the received DMT symbols. That is because retransmission is not requested when the number of erasures is not large enough. At the same time, since fewer number of retransmissions are required, the average retransmission delay would be reduced when $\beta$ increases.

As $\beta$ ranges from 0.6 to 1 , the miss-detection probability increases from $0.3 \%$ to $3 \%$. To achieve the most reliable detection for corrupted symbol, the selection of $0.6 \leq \beta \leq 1$ can be rejected for both criteria. It is because the higher miss-detection probability leads to relatively higher probability of errors transmitting to the receiver.

When $0.12 \leq \beta \leq 0.5$, the miss-detection probability and the average retransmission delay have the stable values. Indeed, $P_{M-D}$ is around $0.3 \%$ and average retransmission delay is around $0.0145 \mathrm{~ms}$. Therefore, we can choose $\beta$ from this range to achieve the reliable and efficient performance. In erasure marking technique, the error correction capability is $\left\lfloor N_{R S}-K_{R S}\right\rfloor$. In our analysis and simulation, we let $\beta=\left\lfloor N_{R S}-K_{R S}\right\rfloor / N_{R S}$, which is inside the above trustful range. 

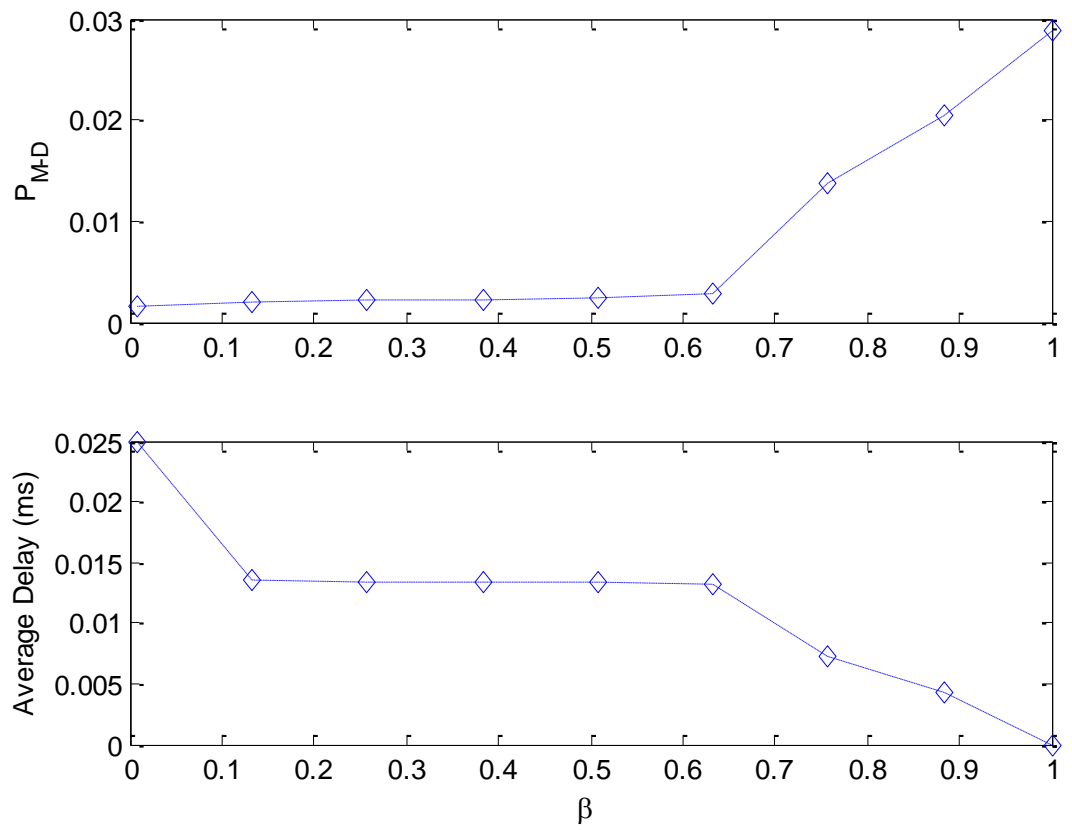

Figure 4.4. Probability of miss-detection and average retransmission delay versus threshold $\boldsymbol{\beta}$ for Criterion B-1
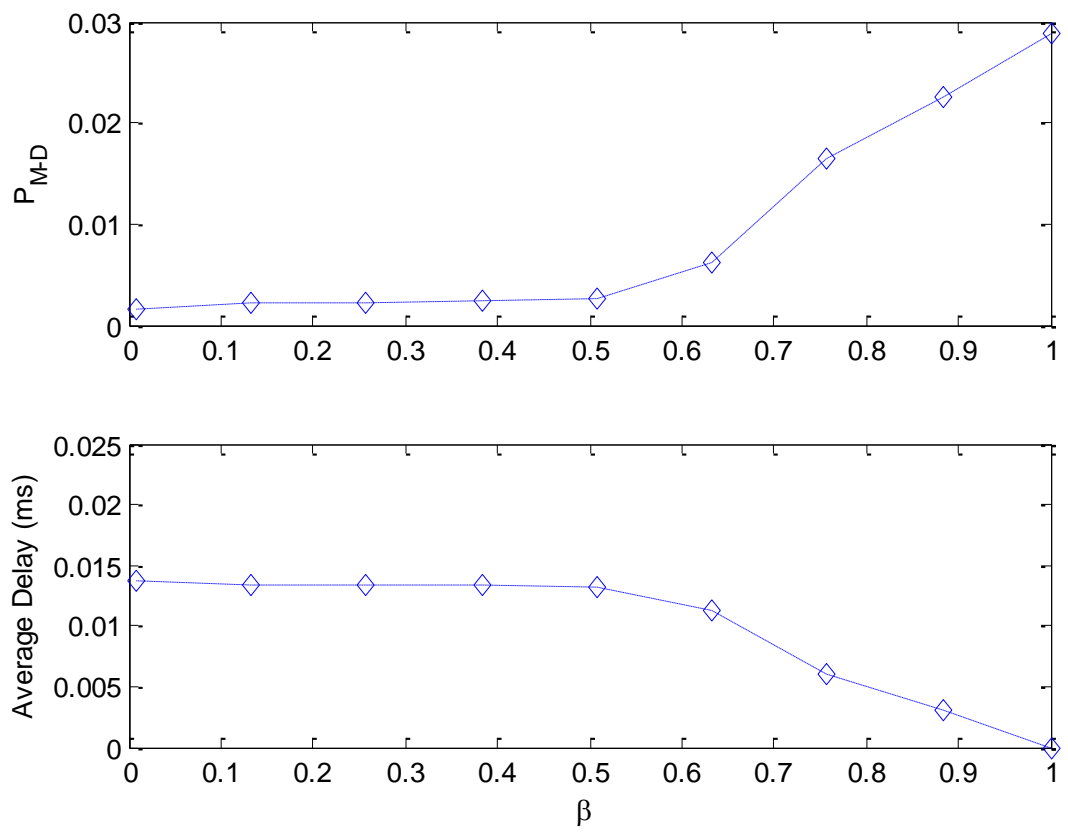

Figure4.5. Probability of miss-detection and average retransmission delay versus threshold $\boldsymbol{\beta}$ for Criterion B-2 
With the selected thresholds $\gamma$ and $\beta$, we can compute the probability of $v$-times retransmission and the delay. As the results are similar for both criteria, in Figure 4.6 we only show the results for Criterion B-1, where the probability of one-time retransmission and average retransmission delay versus IN PSD from the derivation and simulation are compared. When IN with a PSD in the range from -105 to $-100 \mathrm{dBm} / \mathrm{Hz}$, the noise is not strong enough to cause any retransmission, corresponding to a delay close to zero. When IN PSD is about $-80 \mathrm{dBm} / \mathrm{Hz}$ or higher, around $2.8 \%$ of the DMT symbols are corrupted and require one-time retransmission. This corresponds to an average retransmission delay of $0.0145 \mathrm{~ms}$.

In comparison with Criterion A presented in Section 4.1.1, the results of delay are similar for both criteria for most of the IN PSD levels. However, there are some differences. When IN PSD is higher than $-100 \mathrm{dBm} / \mathrm{Hz}$ and lower than $-80 \mathrm{dBm} / \mathrm{Hz}$, there is more chance for Criterion B to call for retransmission, thus the delay is a little higher than Criteron A "Decoding Status". For example, when IN PSD is $-90 \mathrm{dBm} / \mathrm{Hz}$, around $2.1 \%$ of DMT symbols request for retransmission, causing $0.01 \mathrm{~ms}$ average delay. For Criterion A, some IN-corruption samples are successfully corrected by RS decoder so that fewer retransmissions are requested in this IN power range. However, for Criterion B, we only observe the erased samples to make retransmission decision; hence, some symbols that can be corrected by RS-decoder are also requested for retransmission. 

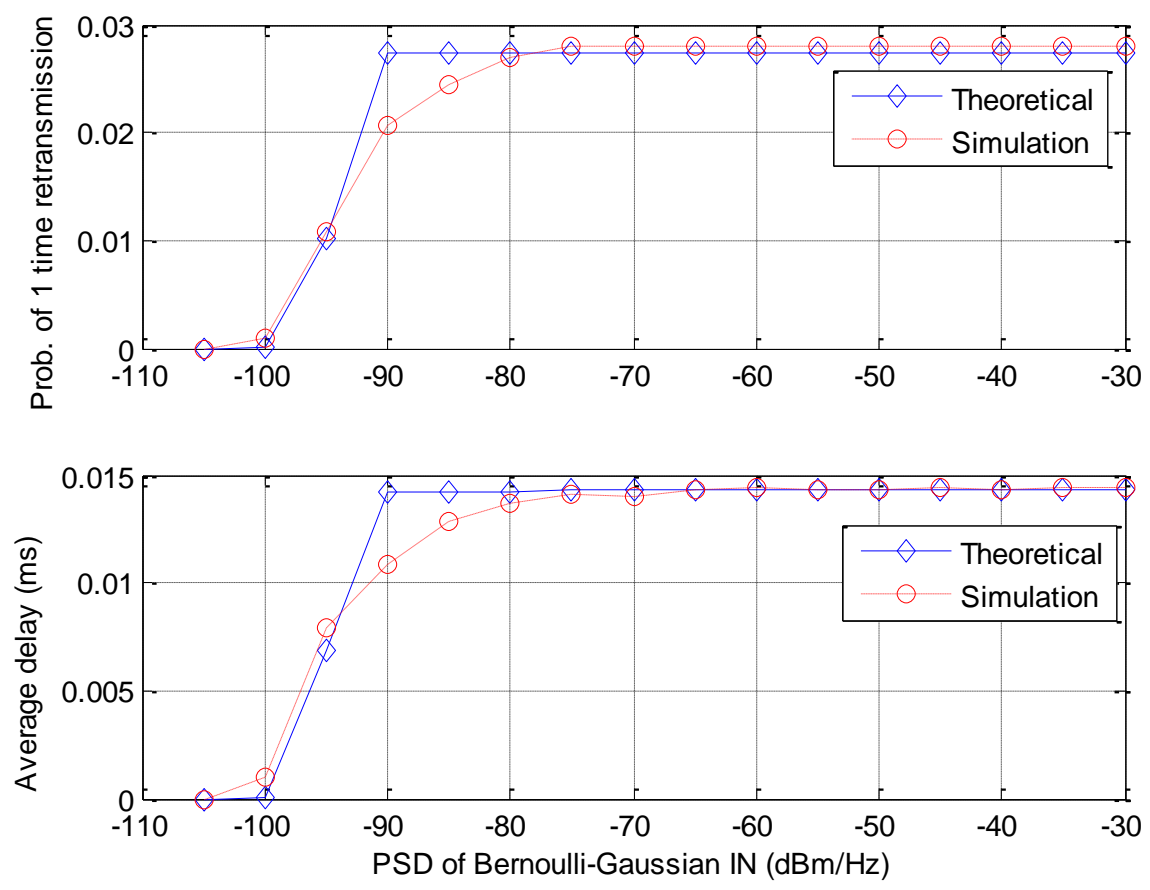

Figure 4.6. One-time retransmission probability $\left(P_{v}, v=2\right)$ and average delay $\left(T_{a v g}\right)$ versus IN PSD for Criterion B “Erasure Marking”

\subsubsection{Performance of Criterion C-'Unused' Tone Status}

For Criterion C, we first investigate the false alarm, miss-detection and delay results when selecting different power threshold $\gamma_{u}$. We find that the false alarm cannot be observed for any $\gamma_{u} \geq 1 \mathrm{~dB}$ tested (i.e., with a very small probability). The reason is that without IN the chance for noise power being greater than the AWGN power plus $\gamma_{u}$ is extremely small. Figure 4.7 shows that the miss-detection probability increases with respect to the growth of $\gamma_{u}$. A larger $\gamma_{u}$ indicates lower probability that noise power exceeds the threshold. Therefore, the chance that some DMT symbols are considered to be not affected by IN is higher. At the same time, fewer retransmission requests are needed in the system and, hence, the average delay decreases when $\gamma_{u}$ grows. To achieve a very short average delay, one may set $\gamma_{u}$ as high as possible. However, the reliability of IN tracking is reduced in such a case, which means that the BER is likely 
to be larger than zero. We trust $\gamma_{u}$ around the target SNR margin (i.e., 6dB) to be a good threshold.
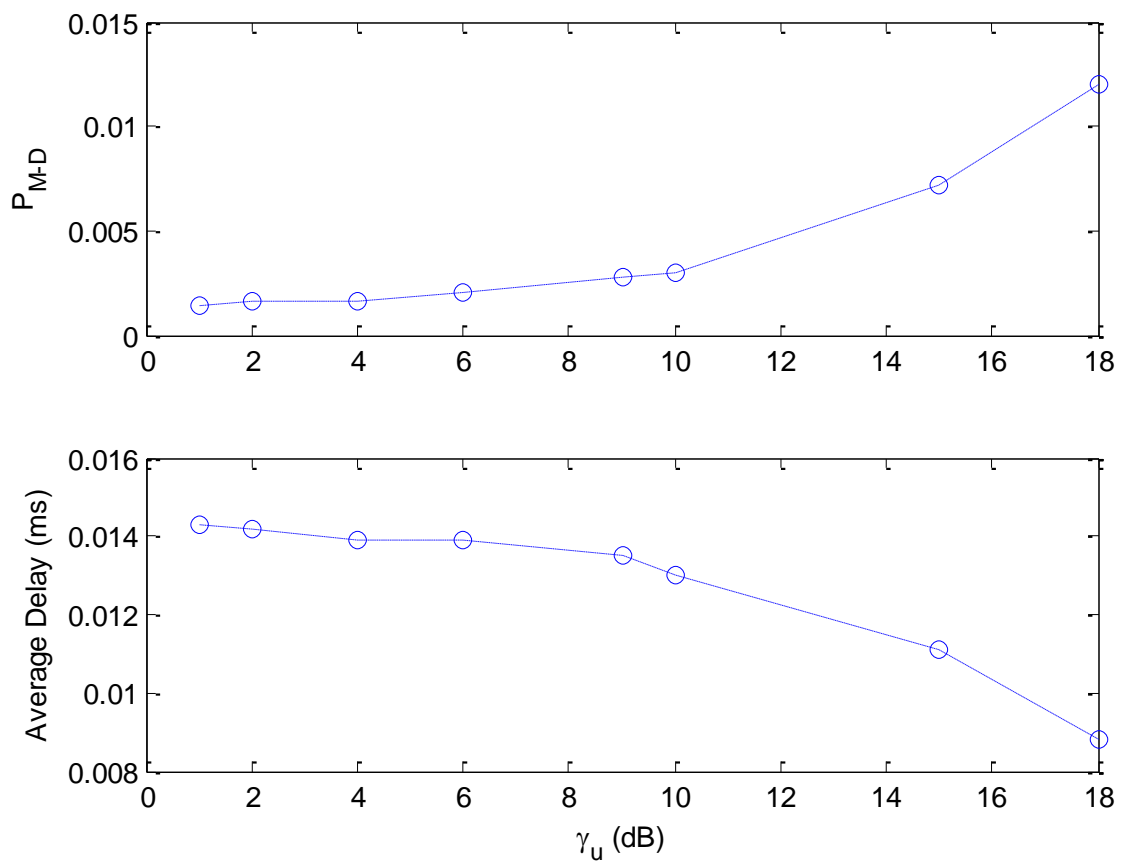

Figure 4.7. Probability of miss-detection and average retransmission delay versus

$$
\gamma_{u}
$$

We choose $\gamma_{u}=6 \mathrm{~dB}$ in the following simulation results. Figure 4.8 shows the probability of one-time retransmission and average delay versus IN PSD. For IN with a PSD less than $-100 \mathrm{dBm} / \mathrm{Hz}$, the noise is not strong enough to cause any retransmission, corresponding to a delay close to zero. The average retransmission delay is around $0.012 \mathrm{~ms}$ when the noise PSD is $-90 \mathrm{dBm} / \mathrm{Hz}$. When IN PSD is about $-85 \mathrm{dBm} / \mathrm{Hz}$ or higher, around $2.8 \%$ of the DMT symbols are corrupted and require one-time retransmission. This corresponds to an average retransmission delay of $0.0145 \mathrm{~ms}$. 

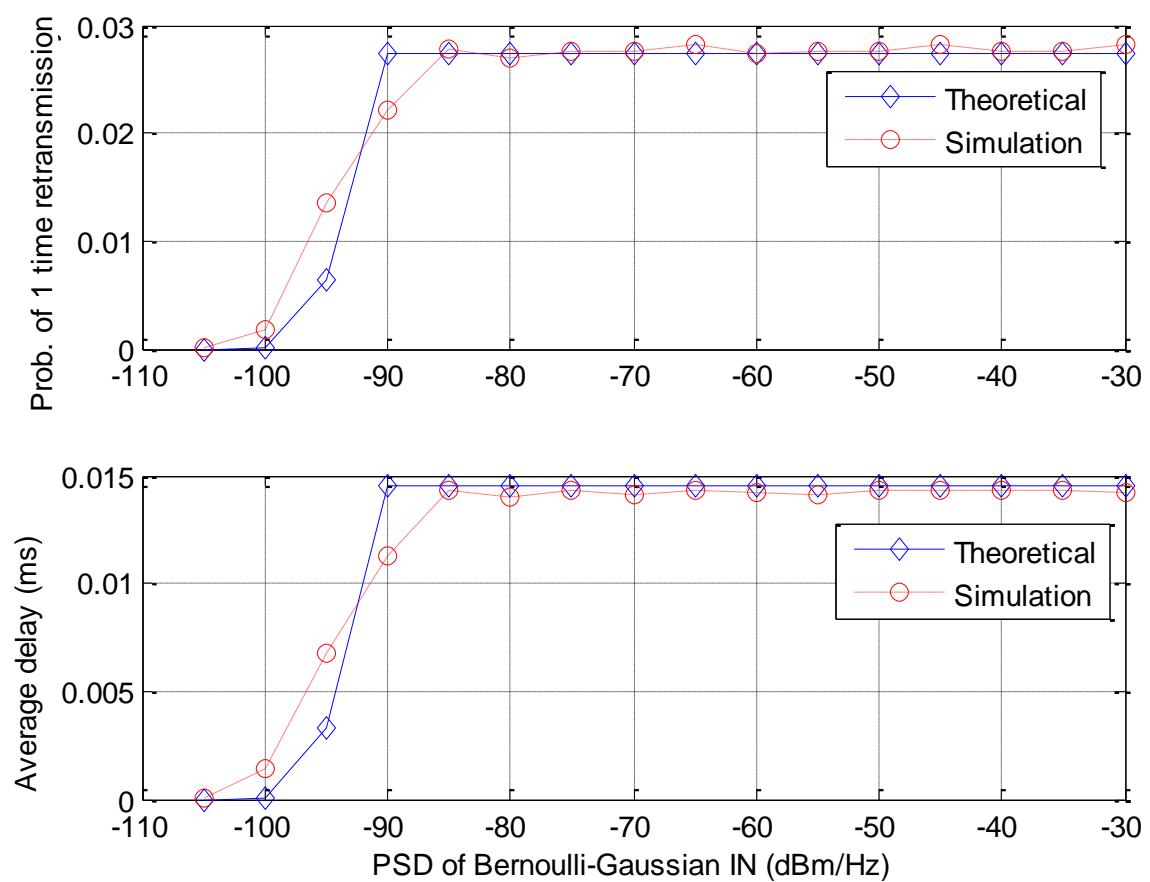

Figure 4.8. One-time retransmission probability $\left(P_{v}, v=2\right)$ and average delay $\left(T_{\text {avg }}\right)$ versus IN PSD for Criterion C "“Unused" Tone Status"

For Criterion C-2, we also select $\gamma_{u}=6 \mathrm{~dB}$. We then need to select $N_{u}$. From Figure 4.9 , it is clear that when $N_{u}$ is larger than $10 \%$ of the number of unused tones, $P_{F-A}$ (the false alarm probability) is 0 . When the threshold $N_{u} / N_{\text {un-tones }}$ increases from 0.03 to 0.95 , miss-detection probability increases from $1.8 \times 10^{-3}$ to $3.8 \times 10^{-3}$, and the average retransmission delay decreases from $0.0148 \mathrm{~ms}$ to $0.0134 \mathrm{~ms}$. The proper choice is therefore to minimize the probability of false alarm, to reduce the average delay as low as possible while ensuring a sufficiently small BER. 

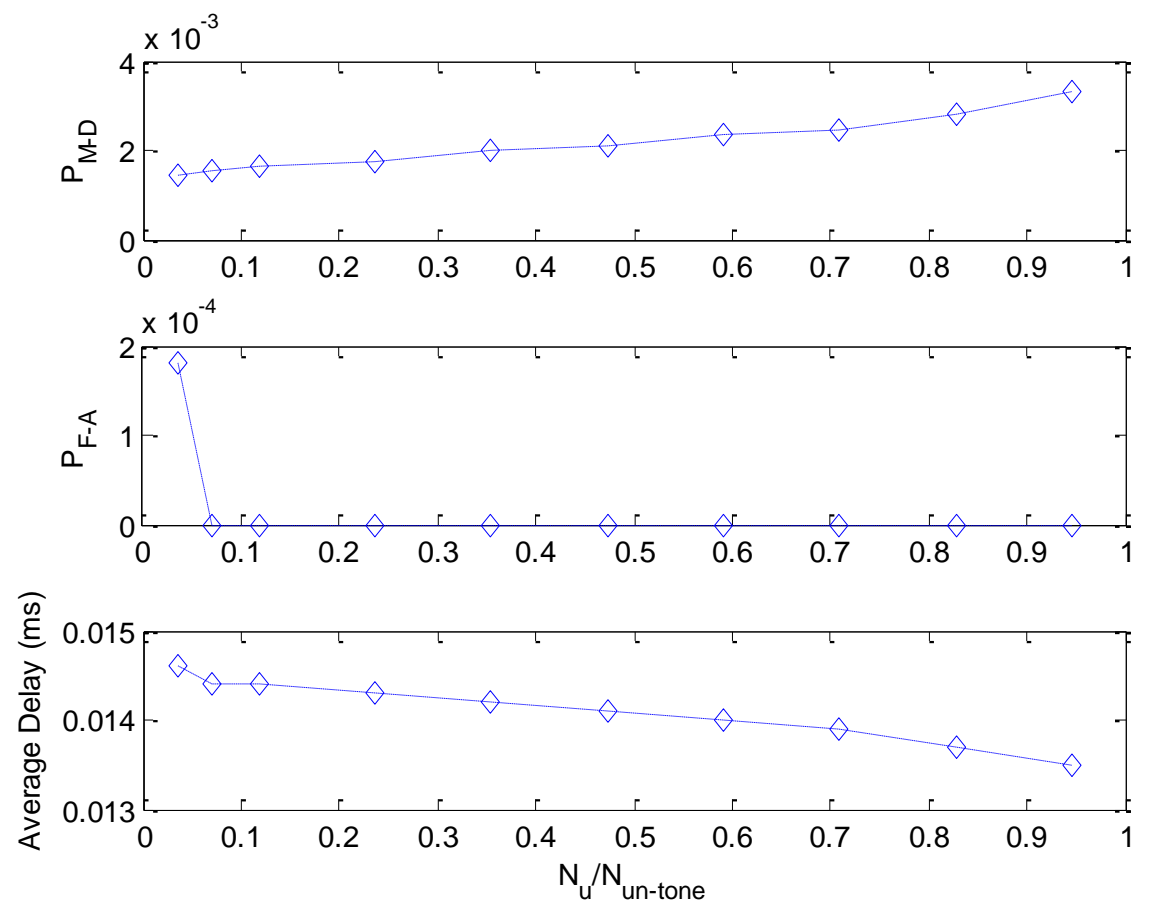

Figure 4.9. Probability of miss-detection, false alarm and average retransmission delay versus $N_{u} / N_{\text {un-tones }}$

In the simulation, the variation of $N_{u}$ gives us a more flexible option to adjust the performance. For more precise IN detection, $N_{u}$ can be as low as $0.06 N_{u n-t o n e s}$; and to achieve a shorter delay, $N_{u}=0.9 N_{\text {un-tones }}$ can be a better choice. In our simulation, we choose $N_{u}=0.3 N_{\text {un-tones }}$ as an example to show the performance of Criterion C-2; and we find that Criterion $\mathrm{C}-2$ has similar performance (probability of one-time retransmission, average retransmission delay etc.) as compared to Criterion C-1.

\subsubsection{BER performance}

Using the derivations in Chapter 3, the residual BER due to the effect of BernoulliGaussian IN for an allowable maximum number of retransmissions versus IN PSD is computed and plotted in Figure 4.10. Simulation results are also included and show a good agreement with the analytical results. Without retransmission, the achieved BER $\left(P_{b a f t, i}\right)$ is as high as $3 \times 10^{-2}$ in the presence of IN with PSD of $-80 \mathrm{dBm} / \mathrm{Hz}$ or higher. 
For IN PSD less than $-100 \mathrm{dBm} / \mathrm{Hz}$, since for most chance only one or two samples of IN corrupts the DMT symbol, the BER is less than $10^{-7}$.

With retransmission, the BER $\left(P_{b a f t, i} P_{v}^{7}\right)$ is quickly reduced to less than $10^{-4}$ and $10^{-9}$ for a maximum allowable number of retransmissions of 1 and 3, respectively. We can conclude that the retransmission is reliable to keep the BER around zero when we allow more than three retransmissions for IN with high PSD corrupting the transmission.

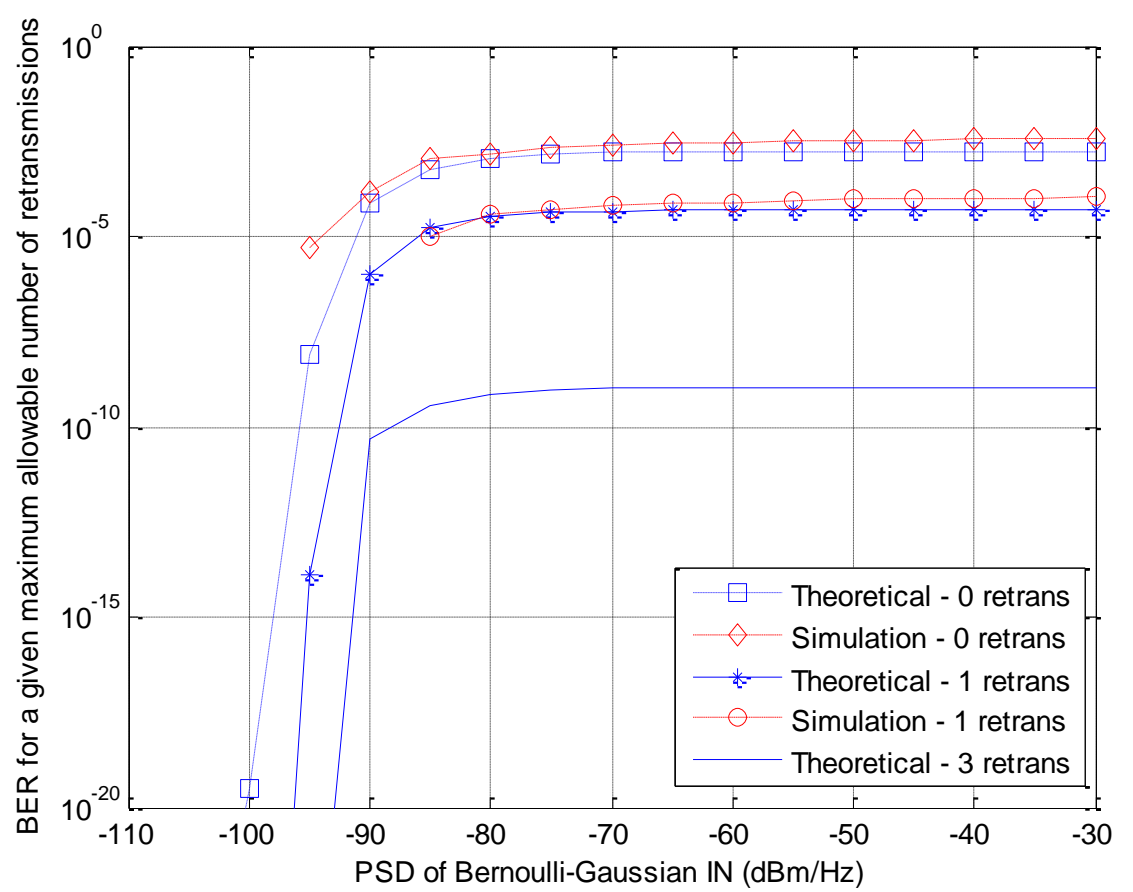

Figure 4.10. BER for a given maximum allowable number of retransmissions

\footnotetext{
${ }^{7} P_{v}$ is taken from Criterion A in this calculation.
} 


\subsection{Simulation Results on Performance for REIN and measured DSL channel}

We also obtained simulation results for a DSL system (as shown in Figures 3.1) with a real channel measured from the 500m 25pair 26AWG cable bundle [29] and REIN with $T=1 /(120 \mathrm{~Hz})$ and $q T=250 \mu s$ [14]. Bit loading for all the tones is performed with the target SNR margin of 9dB, target BER of $10^{-7}$, transmitted signal PSD of $-60 \mathrm{dBm} / \mathrm{Hz}$, and AWGN PSD of $-140 \mathrm{dBm} / \mathrm{Hz}$. As previously discussed, the effect of AWGN is negligible for the settings. However, with REIN of $-116 \mathrm{dBm} / \mathrm{Hz}$ or higher, the provided SNR margin is not sufficiently large to cope with such a high IN power. Table 4.2 summarizes the parameters used in our simulations.

Table 4.2. Simulation parameters

\begin{tabular}{|c|c|c|c|}
\hline Transmitted signal PSD & \multicolumn{3}{|c|}{$-60 \mathrm{dBm} / \mathrm{Hz}$} \\
\hline Target SNR Margin & \multicolumn{3}{|c|}{$9 \mathrm{~dB}$} \\
\hline PSD of AWGN & \multicolumn{3}{|c|}{$-140 \mathrm{dBm} / \mathrm{Hz}$} \\
\hline PSD of REIN & \multicolumn{3}{|c|}{$-116 \mathrm{dBm} / \mathrm{Hz}$} \\
\hline Duration of REIN & \multicolumn{3}{|c|}{$250 \mu \mathrm{s}$} \\
\hline Repetition rate of REIN & \multicolumn{3}{|c|}{$120 \mathrm{~Hz}$} \\
\hline DMT symbol rate & \multicolumn{3}{|c|}{4000 symbols/s } \\
\hline Number of tones & \multicolumn{3}{|c|}{4096} \\
\hline Frequency spacing & \multicolumn{3}{|c|}{$4312.5 \mathrm{~Hz}$} \\
\hline Band plan from Profile 12a [18] & $\begin{array}{c}\text { Band } \\
\text { D1 } \\
\text { D2 } \\
\end{array}$ & $\begin{array}{l}\text { start tone } \\
33 \\
1218 \\
\end{array}$ & $\begin{array}{l}\text { stop tone } \\
857 \\
1959 \\
\end{array}$ \\
\hline$\left(N_{R S}, K_{R S}\right)$ & \multicolumn{3}{|c|}{$(128,112)$} \\
\hline
\end{tabular}

The performance can be evaluated with respect to the delay and BER requirement. Also the miss-detection and false alarm probabilities for the retransmission requests are investigated. 


\subsubsection{Performance for Criterion A - Decoding Status}

Table 4.3. Simulation results for Criterion A and REIN

\begin{tabular}{|c|c|c|c|}
\hline PSD of REIN: dBm/Hz & -116 & -100 & -90 \\
\hline BER without IN & \multicolumn{3}{|c|}{$<10^{-7}$} \\
\hline BER without retransmission (IN) & 0.0039 & 0.0159 & 0.0215 \\
\hline BER with retransmission (IN) & \multicolumn{3}{|c|}{$<10^{-7}$} \\
\hline False alarm Probability & \multicolumn{3}{|c|}{0} \\
\hline Miss-detection Probability & $5.26 \%$ & $2.31 \%$ & $2.16 \%$ \\
\hline Average retransmission delay(ms) & 0.0281 & 0.0289 & 0.029 \\
\hline Maximum retransmission delay (ms) & 0.5 & 0.5 & 0.5 \\
\hline
\end{tabular}

Table 4.3 summarizes the simulation results without and with retransmission technique in the presence or absence of IN. The simulation runs for a total of $2 \times 10^{7}$ bits. Without IN, the obtained BER is zero, which indicates a BER lower than $10^{-7}$ since SNR margin of $9 \mathrm{~dB}$ is enough to ensure the protection under AWGN. When REIN stronger than $-116 \mathrm{dBm} / \mathrm{Hz}$ is present, the obtained $\mathrm{BER}$ of the system without retransmission technique is 0.0039 or higher. It can be concluded that REIN deteriorates the system performance. With retransmission, the obtained BER is 0 for all REIN strengths under consideration.

Table 4.3 also shows the probabilities of the false alarm and miss-detection. The false alarm does not happen in our simulation. It can be explained as follows. Without IN, the chance of unsuccessful decoding is extremely small (since BER $\sim 10^{-22}$ with the SNR margin). For low-strength impulse noise, it is not essential for retransmission request since the decoder can correct transmission errors. On the other hand, the miss-detection probability decreases with the stronger REIN. This is due to the following reasons: i) IN samples are random variables; ii) The RS decoder can correct errors caused by lowpower impulse noise, leading to no decoding failure declared; iii) When impulse power increases, the incorrect decoding takes place more frequently, resulting in low miss- 
detection probability. As a consequence, the decoding status of the decoder can provide more accurate information on the presence of IN with the stronger REIN, avoiding transmission error by retransmitting the IN-corrupted DMT symbols. For low-strength impulse noise, it is not essential to request retransmission since the decoder can correct transmission errors.

Table 4.3 also shows the results of the average retransmission delay (with a buffer size of 16 DTM-symbols), Note that, in DSL systems, the combination of RS coding and interleaving usually requires a delay of $8 \mathrm{~ms}$ to meet INP $=2$ [16]. In the same condition of INP $=2$, as shown in Table 4.3, the retransmission provides the average delay of $0.0281,0.0289$, and $0.029 \mathrm{~ms}$ for REIN PSD of $-116,-100$ and $-90 \mathrm{dBm} / \mathrm{Hz}$, respectively. Also, for all REIN strengths under consideration, the system has a maximum retransmission delay of $0.5 \mathrm{~ms}$. This is because the REIN under investigation has a duration of $250 \mu \mathrm{s}$, corrupting at most two consecutive DMT symbols and causing at most one retransmission for each corrupted DMT symbol. Therefore, the maximum round-trip delay is about $0.75 \mathrm{~ms}$. In other words, the proposed retransmission technique based on decoder failure status considerably reduces the delay as compared to the conventional method using the combination of RS coding and interleaving/deinterleaving.

\subsubsection{Performance for Criterion B - Erasure Marking}

In our simulation, the preliminary test is taken with different thresholds $\gamma$. After comparison, under the condition of different REIN power levels, we assign $\gamma$ to achieve the maximum of $\left(P_{c o r}-P_{f}\right)$ in the first place. The selection of $N_{e}$ is $\left\lfloor N_{R S}-K_{R S}\right\rfloor$ which denotes the error correction capability of error and erasure marking method.

Table 4.4 shows BER with and without retransmission technique in the presence or absence of IN. Both criteria B-1 and B-2 can avoid the bit error with the retransmission procedure. It can be seen that the false alarm does not happen in our simulation. The miss-detection probability slightly decreases with stronger REIN. 
In the same condition of INP $=2$, as shown in Table 4.4, the retransmission provides the average delay of $0.0288,0.0289$, and $0.0289 \mathrm{~ms}$ for the REIN PSD of $-116,-100$ and $-90 \mathrm{dBm} / \mathrm{Hz}$, respectively for Criterion B-1. Very similar average retransmission delays for Criterion B-2 are obtained. The maximum round-trip delay is about $0.75 \mathrm{~ms}$. Hence, the retransmission technique considerably reduces the delay compared to the conventional method. In comparison with Criterion A, the miss-detection probability is lower and the average retransmission delay is somewhat higher here.

Table 4.4. Simulation results for Criterion B and REIN

\begin{tabular}{|c|c|c|c|c|}
\hline \multicolumn{2}{|r|}{ PSD of REIN: $\mathrm{dBm} / \mathrm{Hz}$} & -116 & -100 & -90 \\
\hline \multicolumn{2}{|r|}{ BER without IN } & \multicolumn{3}{|c|}{$<10^{-7}$} \\
\hline \multicolumn{2}{|c|}{ BER without retransmission (IN) } & 0.0039 & 0.0159 & 0.0215 \\
\hline \multicolumn{2}{|c|}{ BER with retransmission (IN) } & \multicolumn{3}{|c|}{$<10^{-7}$} \\
\hline \multirow{4}{*}{ (B1) } & False alarm Probability & 0 & 0 & 0 \\
\hline & Miss-detection Probability & $2.76 \%$ & $2.31 \%$ & $2.31 \%$ \\
\hline & $\begin{array}{c}\text { Average retransmission } \\
\text { delay }(\mathrm{ms})\end{array}$ & 0.0288 & 0.0289 & 0.0289 \\
\hline & $\begin{array}{c}\text { Maximum retransmission } \\
\text { delay }(\mathrm{ms})\end{array}$ & 0.5 & 0.5 & 0.5 \\
\hline \multirow{4}{*}{ (B2) } & False alarm Probability & 0 & 0 & 0 \\
\hline & Miss-detection Probability & $4.21 \%$ & $2.31 \%$ & $2.31 \%$ \\
\hline & $\begin{array}{c}\text { Average retransmission } \\
\text { delay }(\mathrm{ms})\end{array}$ & 0.0284 & 0.0289 & 0.0289 \\
\hline & $\begin{array}{c}\text { Maximum retransmission } \\
\text { delay }(\mathrm{ms})\end{array}$ & 0.5 & 0.5 & 0.5 \\
\hline
\end{tabular}

\subsubsection{Performance for Criterion C -'Unused' Tone Status}

As described in Chapter 3, a threshold $\alpha$ for the noise power on "unused" tones should be selected for the decision of retransmission. If the noise power measured from unused tones exceeds $\alpha$, the presence of IN is declared. We simulate the retransmissionassisted DSL systems with $\gamma_{u}$ ranging from 1 to $16 \mathrm{~dB}$, REIN of $-116 \mathrm{dBm} / \mathrm{Hz}$ and the 
buffer size of 16-DMT symbols. For any $\gamma_{u} \geq 1$, the system does not have any false alarm to report the non-existing IN occupation.
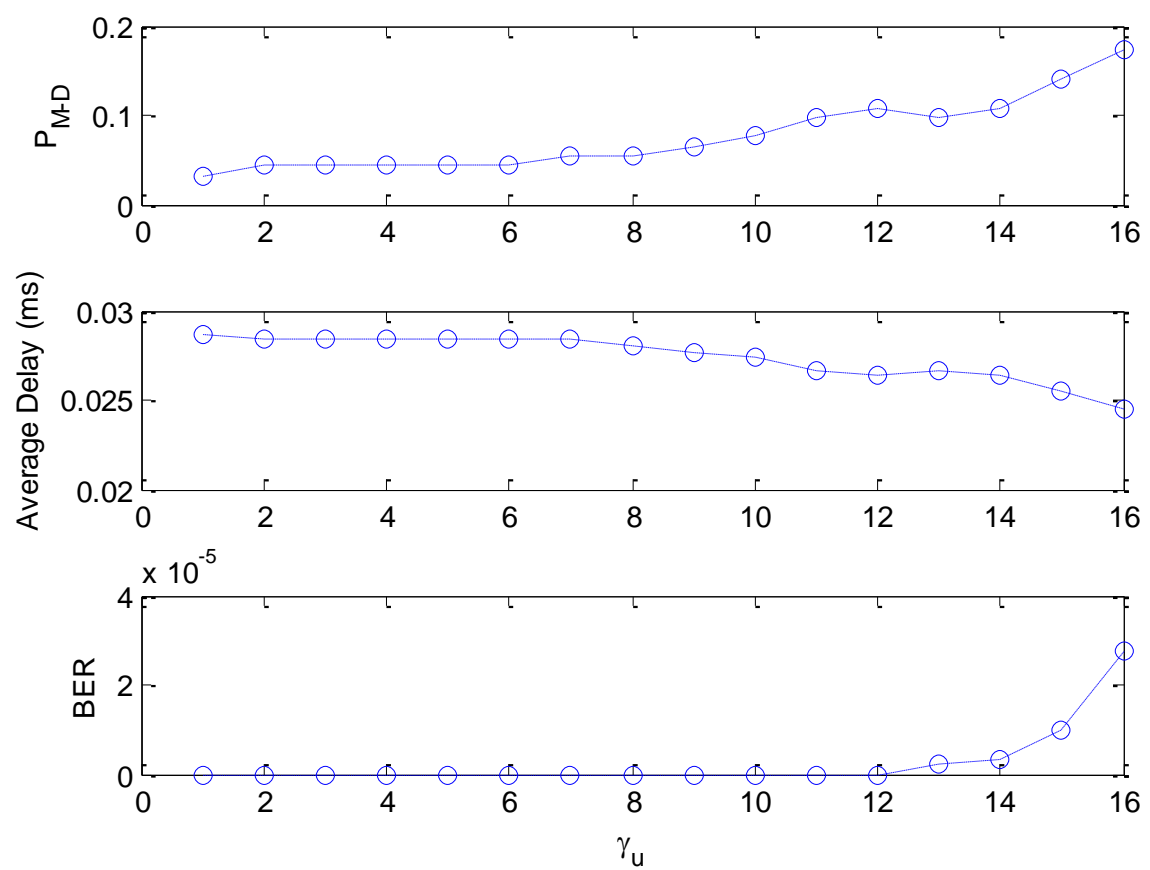

Figure 4.11. Comparison of miss-detection, average retransmission delay and BER versus threshold $\gamma_{u}$

Figure 4.11 illustrates the miss-detection probability, the average retransmission delay and the BER versus threshold $\gamma_{u}$ selection. From this figure, there is a trade-off between BER and the average retransmission delay as predicted in Section 4.1. BER is zero for $\gamma_{u} \leq 12 \mathrm{~dB}$. Therefore, even though SNR margin is just $9 \mathrm{~dB}$, the system is still stable for noise fluctuations up to $12 \mathrm{~dB}^{8}$. However, BER is higher than $10^{-7}$ when $\gamma_{u}>12 \mathrm{~dB}$ and increases with respect to $\gamma_{u}$. It is because the miss-detection probability increases when the noise power threshold is higher; fewer retransmissions are requested, such that the system will accept reception error. Since the average retransmission delay decreases with respect to the increase of $\gamma_{u}$, the appropriate value of $\gamma_{u}$ should be set as high as possible while keeping BER near zero. $\gamma_{u}=\gamma_{m}$ is chosen in our simulation.

\footnotetext{
${ }^{8}$ Perhaps the simulated number of bits generated in our simulation is not enough to observe lower values of BER.
} 
Table 4.5. Simulation results for Criterion $\mathrm{C}$ and REIN

\begin{tabular}{|c|c|c|c|c|}
\hline \multicolumn{2}{|r|}{ PSD of REIN: $\mathrm{dBm} / \mathrm{Hz}$} & -116 & -100 & -90 \\
\hline \multicolumn{2}{|r|}{ BER without IN } & \multicolumn{3}{|c|}{$<10^{-7}$} \\
\hline \multicolumn{2}{|c|}{ BER without retransmission (IN) } & 0.0039 & 0.0159 & 0.0215 \\
\hline \multicolumn{2}{|c|}{ BER with retransmission (IN) } & \multicolumn{3}{|c|}{$<10^{-7}$} \\
\hline \multirow{4}{*}{ (C1) } & False alarm Probability & 0 & 0 & 0 \\
\hline & Miss-detection Probability & $4.38 \%$ & $2.31 \%$ & $2.16 \%$ \\
\hline & $\begin{array}{l}\text { Average retransmission } \\
\text { delay (ms) }\end{array}$ & 0.0283 & 0.0289 & 0.0290 \\
\hline & $\begin{array}{c}\text { Maximum retransmission } \\
\text { delay }(\mathrm{ms})\end{array}$ & 0.5 & 0.5 & 0.5 \\
\hline & & & & \\
\hline \multirow{4}{*}{ (C2) } & False alarm Probability & 0 & 0 & 0 \\
\hline & Miss-detection Probability & $4.11 \%$ & $2.31 \%$ & $2.16 \%$ \\
\hline & $\begin{array}{l}\text { Average retransmission } \\
\text { delay (ms) }\end{array}$ & 0.0284 & 0.0289 & 0.0290 \\
\hline & $\begin{array}{c}\text { Maximum retransmission } \\
\text { delay (ms) }\end{array}$ & 0.5 & 0.5 & 0.5 \\
\hline
\end{tabular}

Table 4.5 displays the BER with/without retransmission in the presence/absence of IN for $\gamma_{u}=9 \mathrm{~dB}$ and different REIN PSDs. As shown in this table, retransmission using IN detection on unused tones helps avoid the transmission error, and thus reduces BER to less than $10^{-7}$ for all REIN power levels under consideration. With the appropriate setting of $\gamma_{u}$ (as high as the target SNR margin), the false alarm probability is zero for both criteria $\mathrm{C}-1$ and $\mathrm{C}-2$. The miss-detection probability slightly decreases with a stronger REIN. The average retransmission delay of about $0.029 \mathrm{~ms}$ and the maximum round-trip delay of $0.75 \mathrm{~ms}$ satisfy the requirement of DSL standards.

We observe that all the three retransmission techniques discussed in this work meet the DSL standards. Criterion $\mathrm{C}$ has the advantage that the retransmission request can be issued more quickly before decoding. However, the prerequisite of Criterion $\mathrm{C}$ is the availability of unused tones. Compared with Criteria B and C, Criterion A is sufficiently reliable and does not need any pre-test to select the optimum threshold. 


\subsection{PSNR Performance of Video transmission over DSL}

Emerging video services offer new business opportunity for telephone companies. In order to bring the satisfaction to the users, the protection of video quality and the reduction of latency are among the main concerns for current DSL techniques. In this section, we evaluate the performance of video transmission over DSL in presence of REIN using retransmission technique.

Peak signal-to-noise ratio (PSNR) test is a useful benchmark for the evaluation of video quality [25]. It is defined as the ratio (in $\mathrm{dB}$ ) of the peak signal energy to the Mean Squared Error (MSE) observed between the processed video sequence versus the original one. The higher PSNR normally indicates a better quality for the video reconstruction. For video with samples $I(i, j)$ and recovered samples $K(i, j)$, of size $m \times n$ (height $\times$ width), the MSE is given by

$$
M S E=\frac{1}{m n} \sum_{i=0}^{m-1} \sum_{j=0}^{n-1}(I(i, j)-K(i, j))^{2},
$$

whereas the PSNR is defined as

$$
P S N R=10 \log _{10}\left(\frac{M A X_{I}^{2}}{M S E}\right) .
$$

Here $M A X_{I}$ is the maximum possible pixel value of the video. For the pixels presented using $B_{p}$ bits per sample, $M A X_{I}$ is $2^{B_{p}}-1$ (e.g., $B_{p}=8$ bits per sample pixels leads to maximum pixel value of 255).

A typical "good" PSNR is around $35 \mathrm{~dB}$ whereas PSNR values of less than $20 \mathrm{~dB}$ are unacceptable [30]. In this section, we will test the PSNR for the cases with or without IN contaminated, and with or without the retransmission technique. Also we present the latency induced by the protection.

The video is transmitted by the following procedure: the video stream is partitioned into three partitions $\mathrm{A}, \mathrm{B}$ and $\mathrm{C}$. The partition 'A' has very high protection to avoid 
decoder failure, and it is transmitted without any error. Partitions B and C both go through the noise-contaminated channel. The video partition encoded by Scalable Video Codec (SVC), is converted into bit stream. The bit streams pass through the DSL system with the condition of with/without IN contamination and with/without retransmission procedure. At the receiver, the bit stream is SVC-decoded and compare to the original video to calculate the PSNR. The retransmission is performed with Criterion A - Decoding Status, since it is a reliable criterion to address the retransmission as shown previously.

Table 4.6 presents the simulation results. Specifically, PSNR is around $47 \mathrm{~dB}$ when the video data is only affected by the background noise. Here, the video can be well recovered at the receiver side. When REIN (with the PSD of $-116 \mathrm{dBm} / \mathrm{Hz}$, duration of $250 \mu \mathrm{s}, 3 \%$ occupation rate) corrupts the video, the PSNR is reduced to $17.4 \mathrm{~dB}$ without any retransmission, resulting in a poor reception. As soon as retransmission is performed, the video quality arises to PSNR of $46 \mathrm{~dB}$, making the system performance very close to that of AWGN-only channel.

Table 4.6. PSNR of video transmission with/without REIN, with/without retransmission

\begin{tabular}{|c|c|c|}
\hline Video transmitted conditions & PSNR $(\mathrm{dB})$ & $\begin{array}{c}\text { Average } \\
\text { retransmission } \\
\text { delay }(\mathrm{ms})\end{array}$ \\
\cline { 1 - 2 } $\begin{array}{c}\text { With effect of AWGN only } \\
\text { (No retransmission) }\end{array}$ & 47.1897 & None \\
\hline $\begin{array}{c}\text { With AWGN+REIN } \\
\text { (No retransmission) }\end{array}$ & 17.4016 & 0.0289 \\
\hline $\begin{array}{c}\text { With AWGN+REIN } \\
\text { (Retransmission applied) }\end{array}$ & 46.1917 & 0.028 \\
\hline
\end{tabular}




\section{Chapter 5}

\section{Conclusions}

\subsection{Thesis Summary}

To protect the DSL transmission against IN, this thesis proposes the retransmission technique instead of interleaving to reduce the delay. Three criteria have been proposed to detect the presence of impulse noise for retransmission request.

For the "Decoding Status" criterion, the probability of retransmission and the average delay are derived for flat fading channel with Bernoulli-Gaussian IN model. The probability that the decoder corrects the DMT symbol wrongly is lower than $10^{-58}$ so that "Decoding Status" criterion is considered to be trustful. In the analysis of the "Erasure Marking" criterion, the main concern is on the threshold effect. This criterion takes advantage of the flexible threshold selection, but it also needs the pre-test to achieve the most reliable performance. When the system leaves some tones "unused", the noise power on those free tones can be observed to request retransmission. The effect of varying the power threshold to detect IN-contaminated symbols is analyzed. We find that a proper choice for this threshold is the target SNR margin for operational ease.

All the three criteria can be considered reliable and give similar performances. "Decoding Status" criterion is the preferable choice because it uses of decoder status report without other prerequisite test, and the probability that decoder makes some wrong correction is sufficiently small.

Simulation results for the frequency-selective fading channel based on measured DSL cable bundle and REIN show that retransmission techniques using the three criteria all can achieve a considerably short average retransmission delay of around $0.029 \mathrm{~ms}$ and maximum round-trip delay of $0.75 \mathrm{~ms}$. The applications such as live video transmission require the delay to be less than $8 \mathrm{~ms}$. In essence, the three techniques all meet the DSL 
standard requirements. Furthermore, simulation results on BER are sufficiently small. PSNR performance of video transmission over DSL systems in presence of frequencyselective fading channel and REIN has also been evaluated by simulation. It is observed that the PSNR is reduced dramatically when REIN contaminates the transmission, while retransmission procedure can recover the PSNR to the level of IN-free condition.

\subsection{Suggested Future Work}

The contributions made by this study have shown that the retransmission technique based on the proposed criteria can mitigate the impact of IN. Some investigation would be worthwhile for further study.

First, the performance analysis in Section 3.3 is based on the Bernoulli-Gaussian IN model. Extension of this performance analysis for other IN models, e.g., REIN, can be an interesting research subject.

Second, the video quality is an important aspect of user-oriented video services. In our simulation, PSNR is used as a performance metric to represent the video quality perceived by the users. However, PSNR is only based on the byte-to-byte data comparison without the interpretation of images [31]. Performance evaluation of video transmission over DSL in presence of IN with other figures of merit that can present more precise video quality measurement can be a useful and interesting work. 


\section{REFERENCES}

[1] J. M. Cioffi, et al., "Digital subscriber line," Scholarpedia, 3(8):3995, http://www.scholarpedia.org/article/Digital_subscriber_line_(DSL), October 21, 2011.

[2] American National Standard Institute (ANSI), T1.413-1998, Asymmetric Digital Subscriber Line (ADSL) Metallic Interface, November, 1998.

[3] ITU-T Recommendation G.992.1, Asymmetrical Digital Subscriber Line (ADSL) transceivers, Geneva, 1999.

[4] J. M. Cioffi, "A multicarrier primer", Tutorial, Stanford University, November 1991.

[5] American National Standard Institute (ANSI) T1.417-2003 (Issue 2), Spectrum Management for Loop Transmissions Systems, 2003

[6] ITU-T Recommendation G.992.3, Asymmetrical Digital Subscriber Line transceivers 2 (ADSL2), Geneva, 2005.

[7] J. Miller and A. Mimnaugh, "Quality - the number one obstacle to IPTV adoption," Digital Fountain, March 2008.

[8] R. Boden, "Real-time ARQ protocol for improved Impulsive Noise robustness of DSL systems," 3rd EuroNGI Conference on Next Generation Internet Networks, pp. 40 - 47, 21 - 23, May 2007.

[9] Z. Li, X. Zhu, A. C. Begen and B. Girod, "Forward and retransmitted systematic lossy error protection for IPTV video multicast," in Proc. Packet Video Workshop, Seattle, WA, May 2009.

[10] R. Nilsson, F. Sjoberg, S. K. Wilson, and M. Isaksson, "Combating impulse noise in DMT based VDSL systems," in Proc. Radioveten, Konf. (RVK), (Karlskrona, Sweden), pp.384-388, June 1999. 
[11] D. Toumpakaris, J. M. Cioffi, and D. Gardan, "A square distance-based byteerasure method for reduced-delay protection of DSL systems from non-stationary interference," in 2003 IEEE Global Telecommunications Conference (GLOBECOM 2003), vol. 4, pp. 2114 - 2119, December 2003.

[12] TR-100, “ADSL\ADSL2 plus performance test plan”, DSL Forum Technical Report, March, 2007.

[13] S. V. Vaseghi, Advanced Digital Signal Processing and Noise Reduction, (Chapter on Impulse Noise), 2nd ed., John Wiley \& Sons Ltd., pp. 354 - 360, 2000.

[14] B. Heuvel, "VDSL2 should also withstand 'PEIN' impulse noise," in ETSI STC TM6 meeting, February 2007.

[15] TR-114, "VDSL2 performance test plan," DSL Forum Technical Report, November 2009.

[16] Broadcom Corporation, "Delivering high-quality video in your IPTV deployment," White Paper, April 2009.

[17] D. Toumpakaris, J. M. Cioffi, and D. Gardan, "Reduced-delay protection of DSL sytesms against nonstationary disturbances," IEEE Trans. on Communications, vol. 52, pp. 1927 - 1938, December 2003.

[18] American National Standard Institute (ANSI), T1E1.4-2003-210R5, Very-high Speed Digital Subscriber Lines (VDSL) Metallic Interface, 2003.

[19] kitz.co.uk, "Interleaving explained," http://www.kitz.co.uk/adsl/interleaving.htm, August 08, 2009.

[20] W. Vermillion, "End-to-End DSL Architectures," (Chapter on Cisco DSL manager), Cisco Press Inc., pp. 290-291, 2003.

[21] ITU-T Recommendation G.993.2, Very High Speed Digital Subscriber Line transceivers 2 (VDSL2), Geneva, February 2006. 
[22] J. M. Cioffi, "Chapter 4: Multi-Channel Modulation," Stanford University website, http://www.stanford.edu/group/cioffi/book/chap4.pdf, January 2011.

[23] J. A. C. Bingham, ADSL, VDSL, and Multicarrier Modulation, John Wiley \& Sons, New York, 2000.

[24] M. Ghosh, "Analysis of effect of Impulse Noise on multicarrier and single carrier QAM system”, IEEE Trans. on Communications, vol. 44, pp. 145-147, February 1996.

[25] ITU-T Draft new Rec. J.ra-psnr, Reference Algorithm for Computing Peak Signal to Noise Ration (PSNR) of a Processed Video Sequence with Constant Spatial Shifts and a Constant Delay, Geneva, February 2009.

[26] S. B. Wicker, "Error Control Systems for Digital Communication and Storage," (Chapter on Block Code Performance Analysis), Prentice-Hall Inc., pp. 238 - 251, New Jersey, 1995.

[27] R. J. McEliece and L. Swanson, "On the decoder error probability for ReedSolomon codes," IEEE Trans. on Information Theory, vol. 32, pp. 701-703, September 1986.

[28] K. Cheung, "More on the decoder error probability for Reed-Solomon codes," IEEE Trans. on Information Theory, vol. 35, no. 4, pp. 895-900, July 1989.

[29] R. Morawski, K. Ho-Van, D. Zhang, and Y. Zhao, "xDSL channel responses and quiet line noise: Measurement and results,” McGill University, Tech. Rep. 2009.

[30] H. Zhao, Y. Q. Shi, and N. Ansari. "Hiding data in multimedia streaming over networks," in Proc. $8^{\text {th }}$ Annual Communication Networks and Services Research Conference, 2010.

[31] S. Winkler and P. Mohandas, "The evolution of video quality measurement: From PSNR to Hybrid Metrics," IEEE Trans. on Broadcasting, vol. 54, no. 3, September 2008. 
[32] D. Zhang, K. Ho-Van, and T. Le-Ngoc, "Impulse noise detection techniques for retransmission to reduce delay in DSL systems," IEEE International Conference on Communications, Ottawa, Ontario, 2012. 